\title{
Accessibility of the resources of near Earth space using multi-impulse transfers
}

\author{
Joan-Pau Sanchez ${ }^{*}$ and Colin R. McInnes ${ }^{\dagger}$ \\ Advanced Space Concepts Laboratory, University of Strathclyde, Glasgow, UK
}

\begin{abstract}
Most future concepts for exploration and exploitation of space require a large initial mass in low Earth orbit. Delivering this mass requires overcoming Earth's natural gravity well, which imposes a distinct obstacle to space-faring. An alternative for future space progress is to search for resources in-situ among the near Earth asteroid population. This paper examines the scenario of future utilization of asteroid resources. The near Earth asteroid resources that could be transferred to a bound Earth orbit are determined by integrating the probability of finding asteroids inside the Keplerian orbital element space of the set of transfers with an specific energy smaller than a given threshold. Transfers are defined by a series of impulsive maneuvers and computed using the patched-conic approximation. The results show that even moderately low energy transfers enable access to a large mass of resources.
\end{abstract}

\section{Nomenclature}

$a=$ semi-major axis of an orbit, $\mathrm{AU}$

$b=$ exponent parameter of power law size distribution, 2.354

$C=$ constant parameter of power law size distribution, 942

$\mathrm{D}=$ asteroid diameter, $\mathrm{km}$ or $\mathrm{m}$

$e=$ eccentricity of an orbit

$f=$ portion of material with $\{a, e, i\}$ capturable with an insertion $\Delta v$ lower than a given limit

$\mathrm{H} \quad=$ absolute magnitude

$\mathrm{h}=$ percentage of $\{a, e, i\}$-orbits that can be phased with the Earth with a $\Delta v$ lower than a limit

$i=$ inclination of an orbit, deg

$m \quad=\quad$ mass of the asteroid, $\mathrm{kg}$

$M \quad=\quad$ mean anomaly of an orbit, deg

$\mathrm{M}_{[\mathrm{Du}-\mathrm{Blo}]}=\quad$ total asteroid mass between upper and lower diameter, $\mathrm{kg}$

$\mathrm{MOID}_{\text {cap }}=$ maximum MOID at which a perigee insertion is possible with a given maneuver, $\mathrm{AU}$.

$N=$ number of asteroids

$p=$ semi-latus rectum of an orbit, $\mathrm{AU}$

$\mathrm{P}=$ probability of finding an capturable asteroid within a volume in Keplerian element space $\{a, e, i\}$

$r_{a}=$ apoapsis altitude, $\mathrm{AU}$

$r_{e n c}=$ distance from the Sun of the intersection point, $\mathrm{AU}$

$r_{p}=$ periapsis altitude, $\mathrm{AU}$

$t_{e}=$ time at the encounter or intersection point, $\mathrm{s}$

$t_{m}=$ time of the phasing maneuver, $\mathrm{s}$

$v_{n}^{\text {enc }}=$ normal Cartesian component of the orbital velocity at the intersection point, $\mathrm{AU} / \mathrm{s}$

$v_{r}^{\text {enc }}=$ radial Cartesian component of the orbital velocity at the intersection point, $\mathrm{AU} / \mathrm{s}$

$v_{p l X}=$ asteroid velocity at the Earth plane crossing point, $\mathrm{AU} / \mathrm{s}$

$v_{0}=$ orbital velocity of the asteroid, AU/s

* jpau.sanchez@strath.ac.uk, member AIAA, Research Fellow, Advanced Space Concepts Laboratory, Department of Mechanical Engineering, University of Strathclyde, Glasgow

† colin.mcinnes@strath.ac.uk, member AIAA, Professor, Advanced Space Concepts Laboratory, Department of Mechanical Engineering, University of Strathclyde, Glasgow 


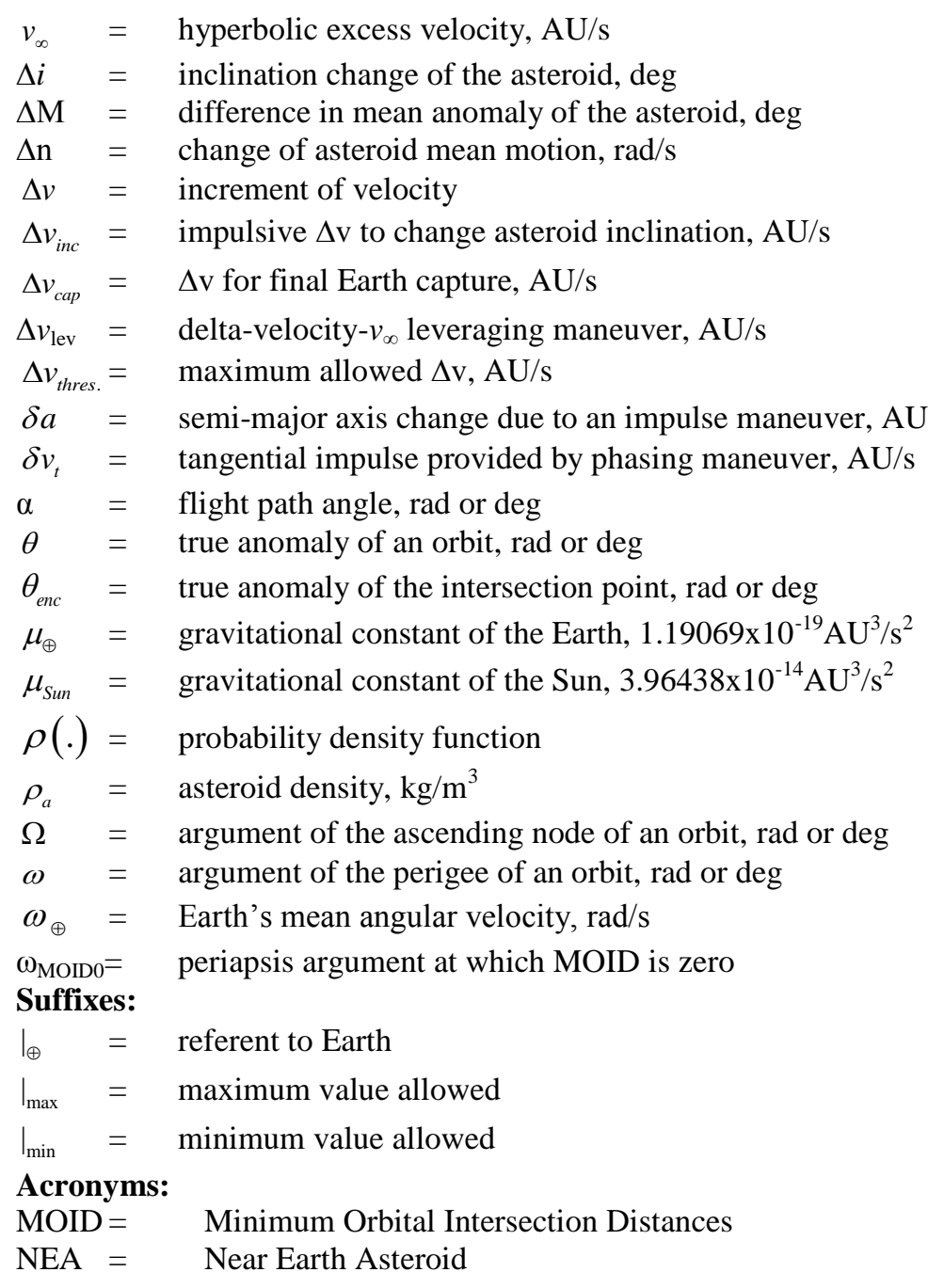

\section{Introduction}

Tost of the plausible futures for human space exploration and exploitation involve a large increase of mass in 1 Earth orbit. Examples include space solar power, space tourism or more visionary human space settlements. Whether this mass is water for crew, propellant for propulsion or materials for structures, these resources will require overcoming Earth's natural gravity well to be delivered in space. Thus, even if technologically possible, this will certainly put a large economic burden to future space progress. An alternative to this approach is to search among the population of asteroids in search of the required reservoir of material ${ }^{1}$.

Asteroids are of importance in uncovering the formation, evolution and composition of the solar system. In particular, near Earth asteroids (NEA) have rose in prominence because of two important points: they are among the easiest celestial bodies to reach from the Earth and they may represent a long-term threat ${ }^{2}$. The growing interest in these objects has translated into an increasing number of missions to NEA, such as the sample return missions Hayabusa ${ }^{3}$ and Marco Polo ${ }^{4}$, impactor missions such as Deep Impact ${ }^{*}$ and possible deflector demonstrator missions such as Don Quixote ${ }^{\dagger}$.

With regard to asteroid deflection, a range of methods have been identified to provide a change in the asteroid linear momentum ${ }^{5}$. Some of these methods, such as the kinetic impactor have been deemed to have a high technology readiness level (TRL), while others may require considerable development. If the capability to impact an asteroid exists (e.g., Deep Impact), or if the capability to deflect an asteroid is available in the near future, a

\footnotetext{
*http://www.nasa.gov/mission_pages/deepimpact/main/index.html

${ }^{\dagger}$ http://www.esa.int/SPECIALS/NEO/SEMZRZNVGJE_0.html
} 
resource-rich asteroid could in principle be maneuvered and captured into a bound Earth orbit through judicious use of orbital dynamics. On the other hand, if direct transfer of the entire NEA is not possible, or necessary, extracted resources could also be transferred to a bound Earth for utilization. It is envisaged that NEA could also be 'shepherded' into easily accessible orbits to provide future resources.

The main advantage of asteroid resources is that the gravity well from which materials would be extracted is much weaker than that of the Earth or the Moon. Thus, these resources could in principle be placed in a weaklybound Earth orbit for a lower energy cost than material delivered from the surface of the Earth or Moon. The question that arises then is how much near-Earth asteroid material is there which can be captured with a modest investment of energy. This paper will attempt to answer this question by analyzing the volume of Keplerian orbital element space from which Earth can be reached under a certain energy threshold and then mapping this analysis to the existing near Earth asteroid population. The resulting resource map provides an accurate assessment of the real material resources of near Earth space as a function of energy investment. It will be shown that there are substantial materials resources available at low energy based on the statistical distribution of near Earth asteroids.

The population of near-Earth objects is modeled in this paper by means of an object size distribution together with an orbital elements distribution function. The size distribution is defined via a power law relationship between the asteroid diameter and the total number of asteroids with size lower than this diameter ${ }^{6}$. On the other hand, the orbital distribution used in this paper will rely on Bottke $e t a .^{7}$ asteroid dynamic model to estimate the probability to find an object with a given set of Keplerian elements.

The dynamic model used to study the Keplerian orbital element space $\{a, e, i\}$ of asteroid-to-Earth transfers assumes a circular Earth orbit with a 1 AU semi-major axis. The Sun is the central body for the motion of the asteroid, and the Earth's gravity is only considered when the NEA motion is in close proximity. Since the orbital transfers will be modeled as a series of impulsive changes of velocity, for some conditions, analytical formulae relate the total change of velocity with the region of Keplerian space that can be reached. If no analytical formulae were found, a root finding algorithm is used to delimit the hyper-volume of Keplerian space that is feasibly exploited under a given delta-velocity budget.

Three different transfer models were included in this paper. First, a phase-free fully analytical two-impulse transfer, which is composed by a change of plane maneuver and a perigee capture burn at Earth encounter. This transfer, like with a Hohmann transfer analysis, provides a good conservative estimation of the exploitable asteroid material. Second, a phase-free analytical one-impulse transfer, which only considers a perigee capture burn during the Earth fly-by. In this second case, only orbits that have initially very low Minimum Orbital Intersection Distances (MOID) can be captured. The MOID is the minimum possible distance between the Earth and the asteroid considering free-phasing for both objects. Finally, three impulses will also be considered as a semi-analytical transfer, allowing us reaching asteroid material that is not Earth-crossing, as well as, reducing the cost of the Earth insertion maneuver using the delta-velocity- $v_{\infty}$ leveraging technique ${ }^{8}$.

\section{Near-Earth Asteroid Model}

In order to determine the resource availability for future asteroid exploitation, a reliable statistical model of the near Earth asteroid population is required. The following section describes an asteroid model of the fidelity required for the subsequent analysis. The asteroid model described is composed of two parts; a size population model, which describes the net number of asteroids as a function of object size and an orbit distribution model that describes the likelihood that an asteroid will be found in a given region of orbital element space.

\section{A. Near Earth Asteroid Population}

The near Earth asteroid population used is taken from the Near-Earth Object Science Definition report ${ }^{6}$. It is based on the results of a substantial number of studies estimating the population of different ranges of object sizes by a number of techniques (see Figure 1 taken from Stokes et al. ${ }^{6}$ ). The Near-Earth Object Science Definition report provides an accumulative population of asteroids that can be expressed as a constant power law distribution function of object diameter as:

$$
N(>D[k m])=C D^{-b}
$$

where $C=942$ and $b=2.354$ (ref.6). This constant power law distribution assumes that the average $1 \mathrm{~km}$ diameter object has an absolute magnitude $\mathrm{H}=17.75$. 


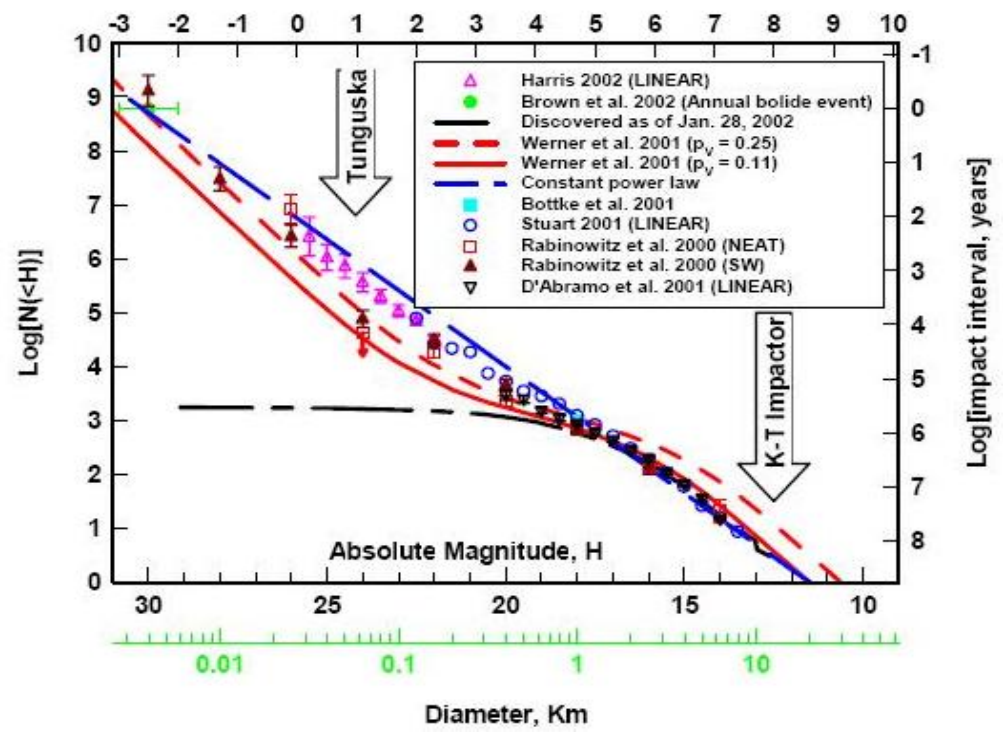

Figure 1. Accumulative size distribution of Near Earth Objects (from Stokes et al. ${ }^{6}$ ).

Assuming a population of asteroids defined by a power law distribution such as Eq.(1), one can easily calculate the total number of objects within an upper and lower diameter:

$$
N\left(D_{\min }>D<D_{\max }\right)=C\left(D_{\min }^{-b}-D_{\max }^{-b}\right)
$$

where $D_{\max }$ and $D_{\min }$ are the maximum and minimum diameter chosen. An estimation of the total asteroid mass composed by all these objects can also be computed. To do so, the following integration needs to be performed:

$$
M_{\left[D_{\max }-D_{\min }\right]}=\int_{N_{D_{\max }}}^{N_{D_{\min }}} m \cdot d N(>D[\mathrm{~km}])
$$

where $m$ is the mass of the asteroid and $N_{D_{\min }}$ and $N_{D_{\max }}$ are the number of objects bigger than $\mathrm{D}_{\min }$ and $\mathrm{D}_{\max }$ respectively.

Assuming that all asteroids have a spherical shape and an average density $\rho_{a}$, the mass $m$ of the asteroid can be defined by $(\pi / 6) \cdot \rho_{a} \cdot D^{3}$ and the integration can be defined as an integration over the asteroid diameter:

$$
M_{\left[D_{\max }-D_{\min }\right]}=\int_{D_{\max }}^{D_{\min }} \frac{\pi}{6} \cdot D^{3} \cdot \rho_{a} \cdot \frac{d N}{d D} d D
$$

where $d N / d D$ is the derivative of Eq. (1) with respect D. Integrating Eq. (4), the total mass of asteroid material composed of asteroids with diameters between $\mathrm{D}_{\max }$ and $\mathrm{D}_{\min }$ results in:

$$
M_{\left[D_{\max }-D_{\min }\right]}=\frac{\pi \cdot \rho_{a} \cdot C \cdot b}{6}\left(\frac{D_{\max }^{3-b}-D_{\min }^{3-b}}{3-b}\right)
$$

The average asteroid density $\rho_{a}$ can be approximated as $2600 \mathrm{~kg} / \mathrm{m}^{3}$ (ref.9). Thus, for example, Eq. (20) can yield the total mass of "Tunguska" size objects (i.e., from $50 \mathrm{~m}$ to $70 \mathrm{~m}$ diameter) in the near Earth space as being in the order of $10^{14} \mathrm{~kg}^{10}$. If the maximum diameter is set equal to the largest near Earth object known, 1036 Ganymed, which is $32 \mathrm{~km}$ in diameter and the minimum object size is set at 1 meters diameter, then Eq. (5) yields a total mass $4.38 \times 10^{16} \mathrm{~kg}$. This should be, approximately, the total mass of asteroid material available in near Earth orbital space. It is still necessary to define the energy requirements for transporting this material to Earth orbit in order to draw conclusions concerning practical resource availability. 


\section{B. Near Earth Asteroid Orbital Distribution}

The present section describes the NEA orbital distribution model used to estimate the likelihood of finding an asteroid within a given volume of Keplerian space $\{\Delta a, \Delta e, \Delta i, \Delta \Omega, \Delta \omega, \Delta M\}$. This likelihood can also be interpreted as the fraction of asteroids within the specified region of the Keplerian space, and thus, if multiplied by Eq.(5), results in the portion of asteroid mass within that region. Hence, the ability of calculating this likelihood, together with the ability of defining the regions of the Keplerian space from which the Earth can be reached with a given $\Delta v$ budget, will later allow us to compute the asteroid resources available in the near-Earth space.

The NEA orbital distribution used here is based on an interpolation from the theoretical distribution model published in Bottke et al. ${ }^{7}$. The data used was very kindly provided by W.F. Bottke (personal communication, 2009). Bottke $e t a l .^{7}$ built an orbital distribution of NEA by propagating in time thousands of test bodies initially located at all the main source regions of asteroids (i.e., the $v_{6}$ resonance, intermediate source Mars-crossers, the 3:1 resonance, the outer main belt, and the transneptunian disk). By using the set of asteroids discovered by Spacewatch at the time, the relative importance of the different asteroid sources could be best-fitted. This procedure yielded a steady state population of near Earth objects from which an orbital distribution as a function of semi-major axis $a$, eccentricity $e$ and inclination $i$ can be interpolated numerically.

The remaining three Keplerian elements, the right ascension of the ascending node $\Omega$, the argument of periapsis $\omega$ and the mean anomaly M, are assumed here uniformly distributed random variables. The ascending node $\Omega$ and the argument of periapsis $\omega$ are generally believed to be uniformly distributed in near Earth orbital space ${ }^{11}$ as a consequence of the fact that the period of the secular evolution of these two angles is expected to be much shorter than the life-span of a near Earth object ${ }^{12}$. Therefore, we can assume that any value of $\Omega$ and $\omega$ is equally possible for any NEA. All values of mean anomaly $M$ are also assumed to be equally possible, and thus $M$ is also uniformly distributed between 0 to $2 \pi$.

A probability density function $\rho(a, e, i)$ has been created by linearly interpolating a 3-dimensional set of data containing the probability density at semi-major axis ranging from 0.05 to $7.35 \mathrm{AU}$ with a partition step size of 0.1 $\mathrm{AU}$, eccentricity ranging from 0.025 to 0.975 with a partition step of 0.05 and inclination ranging from 2.5 to 87.5 deg with a partition step of $5 \mathrm{deg}$. When $\rho(a, e, i)$ requires a value outside the given grid of points (e.g., inclination less than 2.5 degrees) then a nearest neighbor extrapolation is used for the dependence in semi-major axis and eccentricity, while a linear extrapolation is used for the dependence in inclination. Figure 2 shows both the $\rho(a, e, i)$ projected in the $\{a, e\}$ plane and the Aten, Apollo and Amor regions.

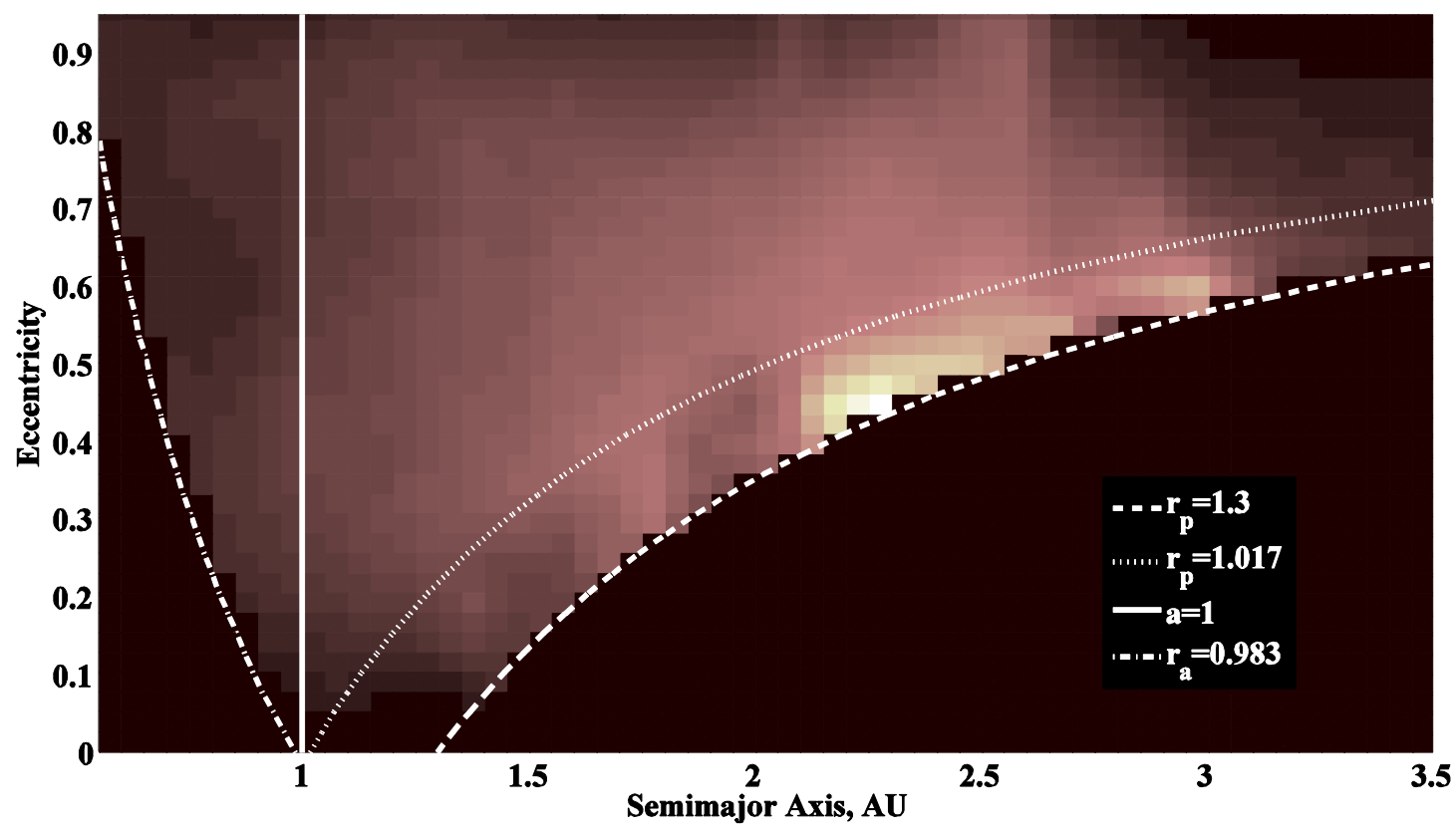

Figure 2. Bottke et al. ${ }^{7} \rho(a, e, i)$ probability distribution built as an interpolation from the model data projected in the $\{a, e\}$ plane. 
Finally, an integration such as:

$$
P=\int_{a_{\min }}^{a_{\max }} \int_{e_{\min }}^{e_{\max }} \int_{i_{\min }}^{i_{\max }} \rho(a, e, i) \cdot d i \cdot d e \cdot d a
$$

yields the probability to find an asteroid within the Keplerian elements defined by $\left[a_{\max }, a_{\min }\right],\left[e_{\max }, e_{\min }\right]$ and $\left[i_{\max }, i_{\min }\right]$. Section III will later describe how these limits can be defined as a function of the delta-velocity budget for different transfer types. The integration of a region of the Keplerian space, by means of Eq.(6), and multiplying this result by Eq.(2) provides a good estimation of the number of objects with diameters between $\left[D_{\max }, D_{\min }\right]$ that should be found within that region:

$$
\left\langle N_{\text {expected }}\right\rangle=P\left(\left[a_{\min }, a_{\max }\right],\left[e_{\min }, e_{\max }\right],\left[i_{\min }, i_{\max }\right]\right) \cdot N\left(D_{\min }>D<D_{\max }\right)
$$

When Bottke et al. ${ }^{7}$ model was under development only 1000 near Earth objects were known, by 15th April 2010, about 7000 objects have been surveyed. The NEA distribution model can then be tested by comparing the prediction of Eq.(7) against the real population. When doing so, care must be taken in comparing our expected population only with the fraction of the surveyed population that is complete or almost completed. The census of small asteroids $(<1 \mathrm{~km})$, which is not completed yet, suffers from observational effects (i.e., objects in orbits that can be more easily spotted are first discovered), and this implies that only the census of objects that are almost completed should be compared with the NEA distribution model. Eq.(1) foresees 861 objects bigger than $1 \mathrm{~km}$ diameter, currently there are 847 known objects with $\mathrm{H} \leq 17.75$, i.e., mean absolute magnitude of objects larger than $1 \mathrm{~km}$ diameter. We then regard the survey of objects larger than $1 \mathrm{~km}$ diameter as almost finished, and therefore with little observation bias. Table 1 compares the observed population of asteroids with the expected population of NEA, calculated by means of Eq.(7). The table shows some difference in each one of the Keplerian regions checked, but in general the expected population matches pretty well the observed NEA and the differences can be regarded as statistical deviations from the mean.

\begin{tabular}{|c|c|c|c|c|c|c|}
\hline & \multicolumn{2}{|c|}{ Expected } & \multicolumn{2}{|c|}{ Observed } & \multicolumn{2}{|c|}{ Difference } \\
\hline & $\mathrm{H} \leq 16$ & $\mathrm{H} \leq 17.75$ & $\mathrm{H} \leq 16$ & $\mathrm{H} \leq 17.75$ & $\mathrm{H} \leq 16$ & $\mathrm{H} \leq 17.75$ \\
\hline Total Asteroids & 129 & 861 & 184 & 847 & $-30 \%$ & $\sim$ \\
\hline Low Inclination & & & & & & \\
\hline$<1 \mathrm{deg}$ & $\sim 1$ & $\sim 7$ & 1 & 3 & $0 \%$ & $133 \%$ \\
\hline$<5 \mathrm{deg}$ & $\sim 10$ & $\sim 68$ & 13 & 65 & $-23 \%$ & $5 \%$ \\
\hline$<10 \mathrm{deg}$ & $\sim 31$ & $\sim 205$ & 41 & 213 & $-24 \%$ & $2 \%$ \\
\hline$<20 \mathrm{deg}$ & $\sim 69$ & $\sim 455$ & 84 & 421 & $-18 \%$ & $8 \%$ \\
\hline$<5 \mathrm{~km} / \mathrm{s}$ & & & 0 & 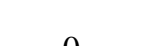 & & \\
\hline$<10 \mathrm{~km} / \mathrm{s}$ & $\sim 0$ & $\sim 0$ & 0 & 0 & $\tilde{\sim}$ & $\tilde{220 \pi}$ \\
\hline$<20 \mathrm{~km} / \mathrm{s}$ & $\sim 32$ & $\sim 211$ & 31 & 177 & $3 \%$ & $19 \%$ \\
\hline
\end{tabular}

Table 1. Comparison between NEA model predictions and surveyed population of asteroids larger than $1 \mathrm{~km}$

\section{Asteroid Material Transfer}

This section will now describe the methodology followed to map the volume of Keplerian space where capture of asteroid resources is deemed feasible. This is the final element, which together with the NEO model described in section II, provides the means to calculate the available asteroid mass for resource exploitation in the near-Earth orbital space. Several transfer types will be considered here, all of them using multi-impulsive trajectories.

The first transfer considered is a two-impulse trajectory. This transfer assumes that intersection with the Earth orbit occurs only if Earth and asteroid are moving on the same plane, and thus, the first maneuver aims to provide the required change on the asteroid orbital plane. With both objects in the same plane, two crossing points are ensured to exist, if orbits actually "cross" (i.e., asteroid perihelion < 1AU and aphelion > 1AU). Assuming also a correct orbital phasing between the two objects, the asteroid would meet the Earth at one of the orbital crossing points. A second maneuver then provides the velocity change necessary to insert the asteroid into a weakly-bound Earth orbit (i.e., parabolic orbit) during the periapsis passage of the Earth fly-by. This simple model is particularly helpful to provide conservative estimates of capturable mass. 
We can also consider the possibility of a single-impulse capture. Even if the asteroid and the Earth have no coplanar motion, asteroid capture would be possible in one single burn if the geometry of the orbits is such that the asteroid's MOID is smaller than the sphere of influence of the Earth. If this geometry occurs one single maneuver provided at the perigee passage may suffice to capture the asteroid in an Earth-bound orbit. Finally, a threeimpulse transfer will also be described. This type of transfer provides access to resources in the non-Earth-crossing Keplerian region and, at the same time, can reduce the $\Delta v$ capture requirement of Earth crossing asteroids. The latter type of maneuver is generally refer as delta-velocity- $v_{\infty}$ leveraging transfer ${ }^{8}$.

All these transfer models will be described as phase-free transfers. This means that the real orbital position is not taken into account, but only the geometry of the orbits is considered. Clearly, in order for an asteroid to meet the Earth during its orbital motion, not only the MOID must be very small (i.e., geometric consideration), but also the position of the Earth and the asteroid inside their mutual orbits must be very precise. Thus, an additional maneuver will be considered in order to provide the gentle push necessary to render the required phasing at the MOID.

\section{A. Two-impulses method}

On this model and in any other transfer model in the paper, it will be assumed that the motion of the asteroid, or any material resources extracted, is dominated by the gravitational influence of the Sun. The Earth is assumed to be in a circular orbit with radius $1 \mathrm{AU}$. When the asteroid has a close encounter with the Earth, the motion will be dominated by the Earth's influence in a patched-conic approximation.

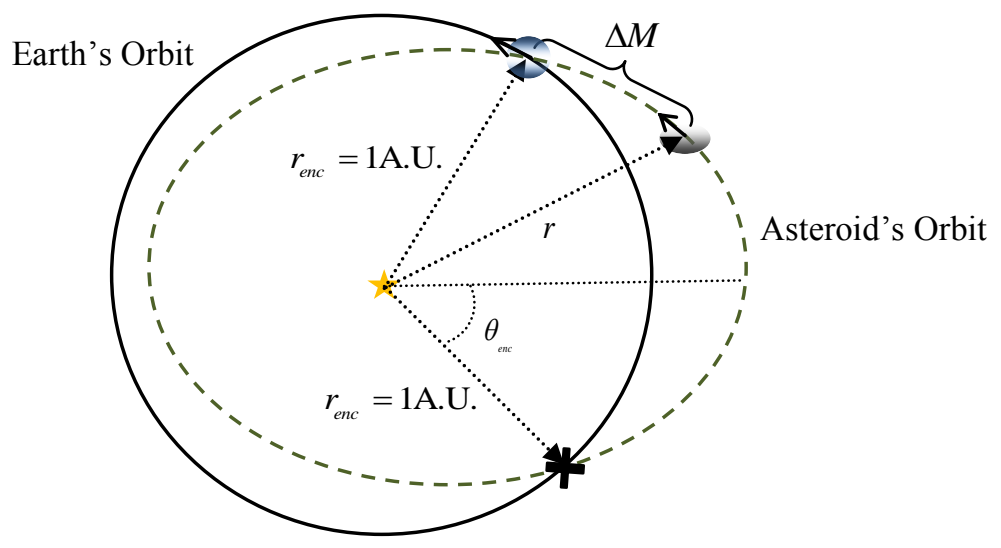

Figure 3. Orbital geometry of the coplanar model.

As shown in Figure 3, an Earth-crossing coplanar asteroid has two intersections (points of MOID equal 0) with the Earth's orbit. These are found when the asteroid is at 1AU from the Sun. Since the distance $r$ from the Sun to the asteroid is known, the equation of the orbit in polar coordinates yields the true anomaly of the two encounters $\theta_{\text {enc }}$ :

$$
\theta_{e n c}= \pm \cos ^{-1}\left(\frac{p-1}{e}\right)
$$

where $p=a\left(1-e^{2}\right)$ is the asteroid's semi-latus rectum and the unit length in Eq.(8), and any of the following formulas in this paper, has been normalized to $1 \mathrm{AU}$.

With the true anomaly of the encounter $\theta_{\text {enc }}$ known, the velocity at the encounter can now be defined by using normal and radial components of a Keplerian orbital motion:

$$
\begin{gathered}
v_{r}^{e n c}=\sqrt{\frac{\mu_{\text {Sun }}}{p}} e \sin \left(\theta_{\text {enc }}\right) \\
v_{n}^{e n c}=\sqrt{\frac{\mu_{\text {Sun }}}{p}}\left(1+e \cos \left(\theta_{\text {enc }}\right)\right)
\end{gathered}
$$

where $v_{r}^{e n c}$ and $v_{n}^{e n c}$ are the radial and normal velocity at the MOID point. Using Eq. (8) and Eqs.(9)-(10), the encounter velocity can be rewritten in a more suitable form: 


$$
\begin{gathered}
v_{r}^{e n c}=\sqrt{\frac{\mu_{\text {Sun }}}{p}\left(e^{2}-(p-1)^{2}\right)} \\
v_{n}^{\text {enc }}=\sqrt{\mu_{\text {Sun }} p}
\end{gathered}
$$

Whenever the Earth-coplanar asteroid meets the Earth at $\theta_{\text {enc }}$, the velocity of the asteroid relative to the Earth will then be $\left(v_{r}^{e n c}, v_{n}^{e n c}-\omega_{\oplus} \cdot r_{e n c}\right)$ with the Earth moving at an angular velocity:

$$
\omega_{\oplus}=\sqrt{\frac{\mu_{\text {Sun }}}{a_{\oplus}^{3}}} \text { or } \omega_{\oplus}=\sqrt{\mu_{\text {Sun }}} \text { since } a_{\oplus}=1 \mathrm{AU}
$$

\section{Change of Plane}

The first impulsive maneuver in this capture sequence provides the change of plane necessary to make the asteroid orbit coplanar with the Earth. Using a more complex and realistic sequence of maneuvers, a single combined maneuver could provide both the required phasing and the change of plane such that an Earth flyby occurs. In that case, the asteroid transfer to Earth would not need to be strictly coplanar with the Earth and the change of velocity necessary for the maneuver would be minimized. Unfortunately, this procedure would require a full numerical optimization for each individual case, which would be unmanageable for the scope of this paper.

A simpler approach is to consider a change of plane maneuver such as:

$$
\Delta v_{i n c}=2 v_{p l X} \cdot \sin \left(\frac{\Delta i}{2}\right)
$$

where $\Delta v_{i n c}$ is the impulsive change of velocity necessary to change the orbital plane by $\Delta \mathrm{i}$, and $v_{p l X}$ is the velocity of the asteroid at the Earth orbit plane crossing. Equation (14) allows a more analytical approach to the problem and at the same time provides a worst case scenario for the cost of the change of plane.

The velocity of the asteroid at the Earth crossing plane $v_{p l X}$ will vary with the geometry and orientation of the asteroid orbit, i.e., $a$ and $e$, and argument of the periapsis $\omega$. As it has been previously discussed, the asteroid population model assumes that the argument of the periapsis $\omega$ behaves as a stochastic variable. As a consequence, and since the purpose of this model is to assess the asteroid resources that could be captured at the Earth, $v_{p I X}$ is defined as the velocity of the asteroid at its semi-latus rectum $p$, which again yields the worst case scenario for the change of plane maneuver for each orbital geometry. By doing so, Eq. (14) becomes a function only of the semimajor axis $a$, eccentricity $e$ and inclination $i$ of the asteroid and can be written as:

$$
\Delta v_{\text {inc }}=2 \sqrt{\frac{\mu_{\text {Sun }}}{p}\left(1+e^{2}\right)} \cdot \sin \left(\frac{\Delta i}{2}\right)
$$

\section{Final Earth Insertion}

If MOID is zero or almost zero, the Earth encounter could be easily tuned by a phasing maneuver so that the altitude during the Earth fly-by is some given minimum distance (chosen here to be 200km) above the Earth's surface. At this minimum altitude a final insertion maneuver could be performed. Clearly, for large NEA there would be an issue of impact hazard to be considered, however for smaller bodies this hazard can be mitigated since bodies of tens of meters of diameter should completely ablate in the atmosphere ${ }^{13}$. Thus, bodies in the order of 10 meters diameter may be considered as perfect targets for first capture demonstrator missions.

A parabolic orbit is assumed here to be the threshold between an Earth-bound orbit and an Earth escape orbit. Hence, the $\Delta v$ necessary for an Earth capture $\Delta v_{\text {cap }}$ at the perigee passage results on:

$$
\Delta v_{c a p}=\sqrt{\frac{2 \mu_{\oplus}}{r_{p}}+v_{\infty}^{2}}-\sqrt{\frac{2 \mu_{\oplus}}{r_{p}}}
$$

where $v_{\infty}$ is the hyperbolic excess velocity and $r_{\mathrm{p}}$ is the pericenter altitude. As described at the beginning of section III.A, the hyperbolic excess velocity is defined by the relative Earth encounter velocity $\left(v_{r}^{e n c}, v_{n}^{e n c}-\omega_{\oplus} \cdot r_{e n c}\right)$ and is therefore:

$$
v_{\infty}^{2}=\frac{\mu_{\text {Sun }}}{p}\left(e^{2}-(p-1)^{2}\right)+\left(\sqrt{\mu_{\text {Sun }} p}-\omega_{\oplus}\right)^{2}
$$


since $r_{\text {enc }}$ is equal to 1 using distance units in astronomical units. Considering that the Earth angular velocity $\omega_{\oplus}$ is $\sqrt{\mu_{\text {Sun }}}$ for a circular orbit of radius of $1 \mathrm{AU}$ and using simple arithmetic manipulation, Eq.(17) can be simplified to:

$$
v_{\infty}^{2}=\mu_{\text {Sun }}(3-1 / a-2 \sqrt{p})
$$

which is in fact the Tisserand Criterion for the case of ecliptic orbits and fly-by distance at 1 AU.

\section{Keplerian Feasible Regions}

Given equations (15) and (16), we can now easily estimate the $\Delta v$ budget necessary to transfer/capture asteroid material to/at Earth from a given $\{a, e, i\}$ region, $\Delta v=\Delta v_{\text {inc }}+\Delta v_{\text {cap }}$. These two equations can be rearranged so that they yield the Keplerian regions within which capture of asteroid resources is ensured to be bellow a given threshold $\Delta v_{\text {threshold}}$. Figure 4 shows the Keplerian region in the plane $\{a, e\}$ where asteroid resources can be transfer to Earth with a total $\Delta v$ equal or lower than $2.37 \mathrm{~km} / \mathrm{s}$. This $\Delta v$ corresponds to the Moon's escape velocity, thus offering a direct comparison between material available at the Moon and within an equivalent energy threshold elsewhere in the solar system. Also, superimposed in the figure are almost 5,000 asteroids (tiny dots and small crosses), which had been surveyed by April 2010.

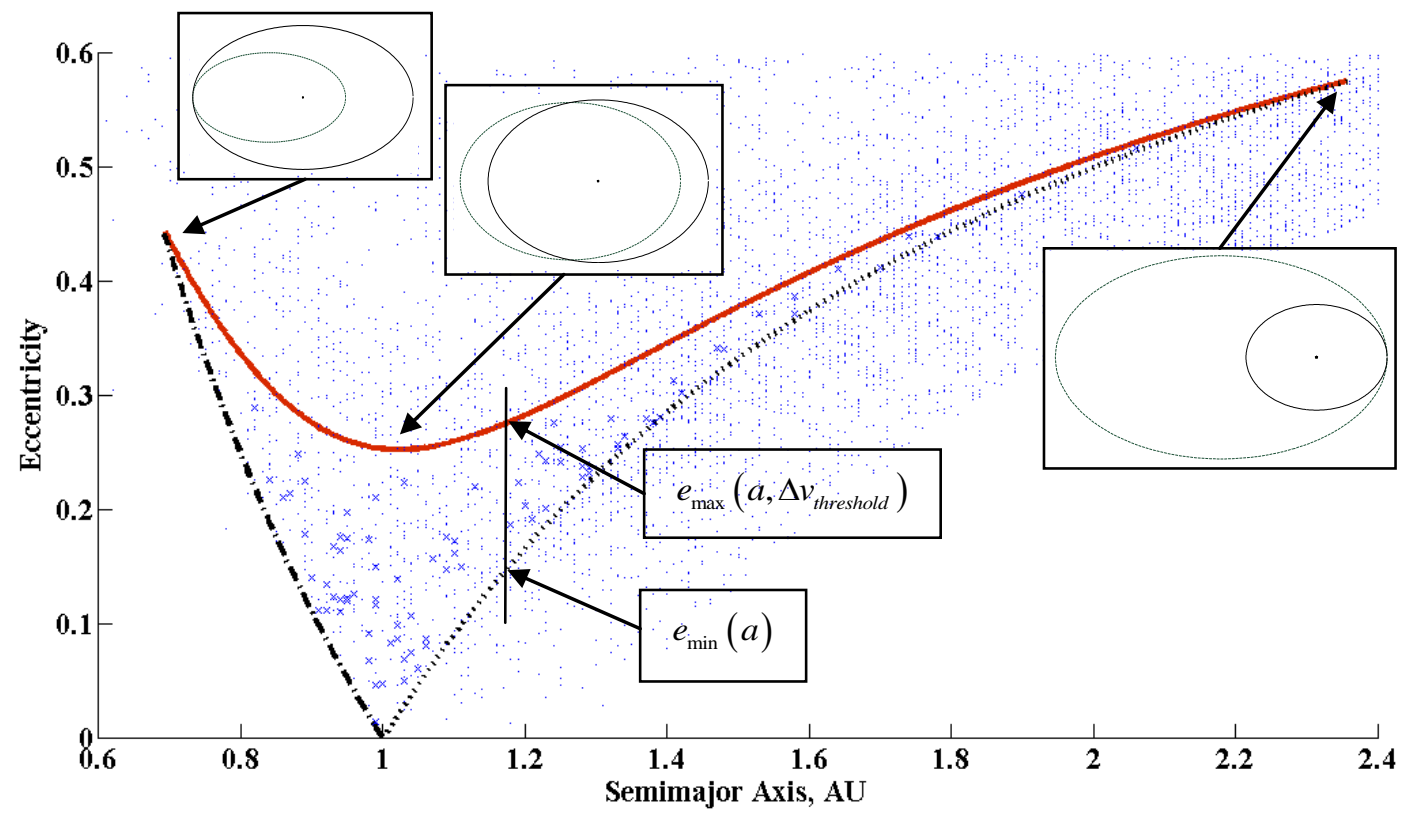

Figure 4. Keplerian \{a,e\} space reached by a maneuver of 2.37 km/s (i.e., Moon's escape velocity). Superimposed are all near Earth asteroids known within the \{a,e\} space as of April 2010.

Figure 4 shows three different lines (solid, dash-dotted and dotted line) delimiting an area in the $\{a, e\}$ plane. The solid line results from expressing Eq.(16) as an explicit function of the semi-major axis $a$ and $\Delta v_{\text {cap }}$ necessary for an Earth capture:

$$
e\left(\Delta v_{\text {cap }}, a\right)=\sqrt{1-\frac{1}{4 a}\left(3-\frac{1}{a}-\frac{v_{\infty}^{2}}{\mu_{\text {sun }}}\right)^{2}} \text { where } v_{\infty}^{2}=\left(\Delta v_{\text {cap }}+\sqrt{\frac{2 \mu_{\oplus}}{r_{p}}}\right)^{2}-\frac{2 \mu_{\oplus}}{r_{p}}
$$

Equation (19) therefore yields the value of eccentricity for which an asteroid with semi-major axis $a$ can be captured with a maneuver $\Delta v_{\text {cap }}$ at the perigee passage. Asteroids with semi-major axis $a$, but eccentricity lower than the result provided by Eq.(19) should be captured with a maneuver lower than $\Delta v_{c a p}$. Thus, if $\Delta v_{c a p}$ is set to the maximum allowed maneuver $\Delta v_{\text {threshold }}$, the eccentricity resulting from Eq. (19) is also the maximum allowed eccentricity, $e_{\max }=e\left(\Delta v_{\text {threshold }}, a\right)$.

Eccentricities lower than $e_{\max }$ require lower $\Delta v$ maneuvers to be captured at the Earth, but there is a geometrical limit to the minimum Earth insertion maneuver $\Delta v_{c a p}$.The minimum $\Delta v_{\text {cap }}$ occurs when the encounter geometry is 
such that the intersection is at the line of apsis. With this geometry only one intersection point exists, and lower eccentricities imply orbits with no Earth crossing points (see Figure 4). The minimum allowed eccentricity for an orbit with semi-major axis $a$ is therefore:

$$
e_{\text {min }}(a)=\left\{\begin{array}{l}
(1-1 / a) \text { if } a \geq 1 \\
(1 / a-1) \text { if } a<1
\end{array}\right.
$$

so that, if $a \geq 1$, the periapis radius is 1 (see dotted line in Figure 4), and, if instead $a<1$, the apoapsis is 1 (see dashdotted line in Figure 4).

Once the analytical expressions for the maximum and minimum eccentricity $e_{\max }$ and $e_{\min }$ are known, the maximum and minimum allowed semi-major axis $a$ can be computed by finding when $e_{\max }\left(\Delta v_{\text {threshold }}, a\right)=e_{\text {min }}(a)$ occurs. The latter equation results in a second degree polynomial with the following two solutions:

$$
a_{\text {min }}\left(\Delta v_{\text {threshold }}\right)=\frac{1}{1-\frac{v_{\infty}^{2}}{\mu_{s}}+2 \sqrt{\frac{v_{\infty}^{2}}{\mu_{s}}}} \text { or } a_{\text {max }}\left(\Delta v_{\text {threshold }}\right)=\frac{1}{1-\frac{v_{\infty}^{2}}{\mu_{s}}-2 \sqrt{\frac{v_{\infty}^{2}}{\mu_{s}}}}
$$

where $v_{\infty}^{2}$ is defined as in Eq.(19).

Inside this delimited area within the $\{a, e\}$ Keplerian space, we can ensure that the coplanar capture maneuvers will be lower than the limit threshold. Thus, the reminder impulse, $\Delta v=\Delta v_{\text {threshold }}-\Delta v_{\text {cap }}(a, e)$, can be used for changing the plane of any available objects as described in section III.A.1. Rearranging Eq.(15), the maximum object's inclination that can be modified and set into a coplanar motion with the Earth results:

$$
i_{\max }\left(a, e, \Delta v_{\text {threshold }}\right)=2 \cdot \sin ^{-1}\left(\frac{\Delta v_{\text {threshold }}-\Delta v_{\text {cap }}(a, e)}{2\left(\frac{\mu_{\text {Sun }}}{p}\left(1+e^{2}\right)\right)^{1 / 2}}\right)
$$

Finally, any object within the Keplerian volume defined by equations (19) to (22) will require a total $\Delta v$ lower than the limit threshold and some $\Delta v$ budget will still be available for phasing. Note that two types of symbols were used in Figure 4 to represent the surveyed asteroids. The small crosses represent all those objects that fall inside the volume calculated by the limits previously described when the Moon's escape velocity is used as $\Delta v_{\text {threshold }}$. Thus, dots falling inside the area delimited in Figure 4 have inclinations larger than the result of equation (22). As shown in the figure, a considerable number of surveyed objects have orbits from which material could be transferred to the Earth with a lower energy requirement than from the surface of the Moon. It is also important to remark that by April 2010 only the survey of objects larger than $1 \mathrm{~km}$ diameter is virtually completed, while less than $0.1 \%$ of the objects between 30 and 300 meters are known. Hence, an important portion of objects are still to be discovered.

\section{B. One-impulse method}

As shown in Figure 3, if the asteroid is coplanar with the Earth orbit, two orbital crossing points will always exist, as long as the periapsis and apoapsis of the asteroid's orbit are smaller and larger than 1 AU, respectively. On the other hand, if the asteroid is not coplanar with the Earth orbit, only specific values of the angle of the periapsis $\omega$ will render an orbital intersection or a MOID small enough for a capture to be possible (see Figure 5). 


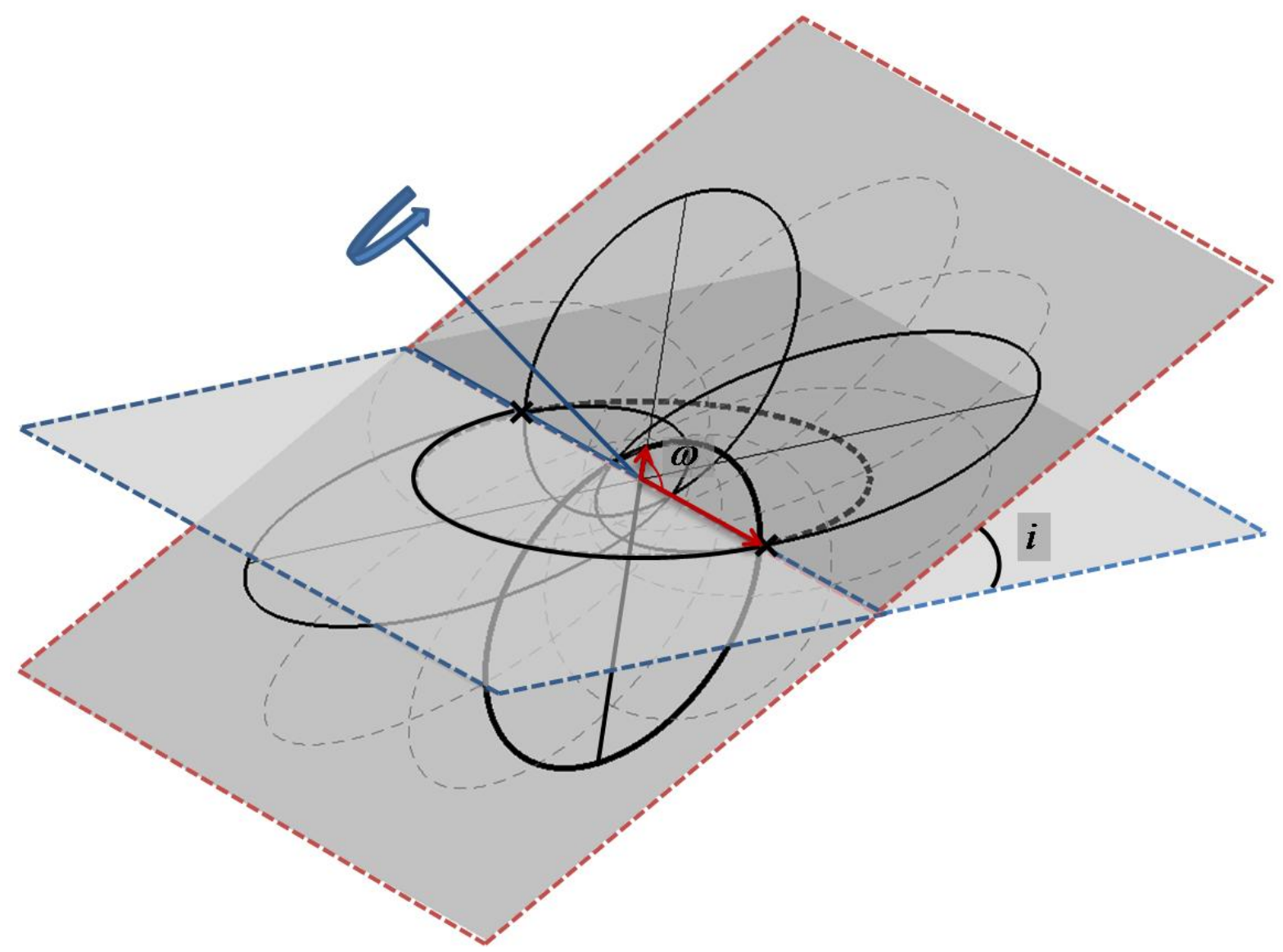

Figure 5. Representation of all possible orientations of an orbit as a function of argument of the periapsis $\omega$. The figure shows two orbital planes, one for the Earth orbit and one for the asteroid orbit. By continuously changing the argument of the periapsis, all possible orientations of the asteroid orbit in the plane are yielded. The two crosses mark the Earth orbital crossing points which are possible only for four different values of the argument of the periapsis $\omega$. Two arrows show the argument of the periapsis $\omega$ for one of the four configurations.

As shown by Figure 5, only 4 specific values of $\omega$ yield a MOID equal to zero (i.e., an intersection between the two orbits). Except if the semilatus rectum $p$ is equal to 1, in which case there will be only two values of $\omega$ yielding two simultaneous crossing points. Equation (8) already provided the two possible true anomalies that give the asteroid a distance of 1AU from the Sun. Therefore, for the orbital intersection to occur in the non-ecliptic asteroid case, one of these two angles is required to coincide with the line of nodes, i.e., the straight line where the two orbital planes meet. This yields four different arguments of the periapsis $\omega$ for which the MOID is 0 :

$$
\omega_{\text {MOID } 0}=\left\{\begin{array}{llll}
\pi-\theta_{\text {enc }} & \theta_{\text {enc }}-\pi & \theta_{\text {enc }} & -\theta_{\text {enc }}
\end{array}\right\}
$$




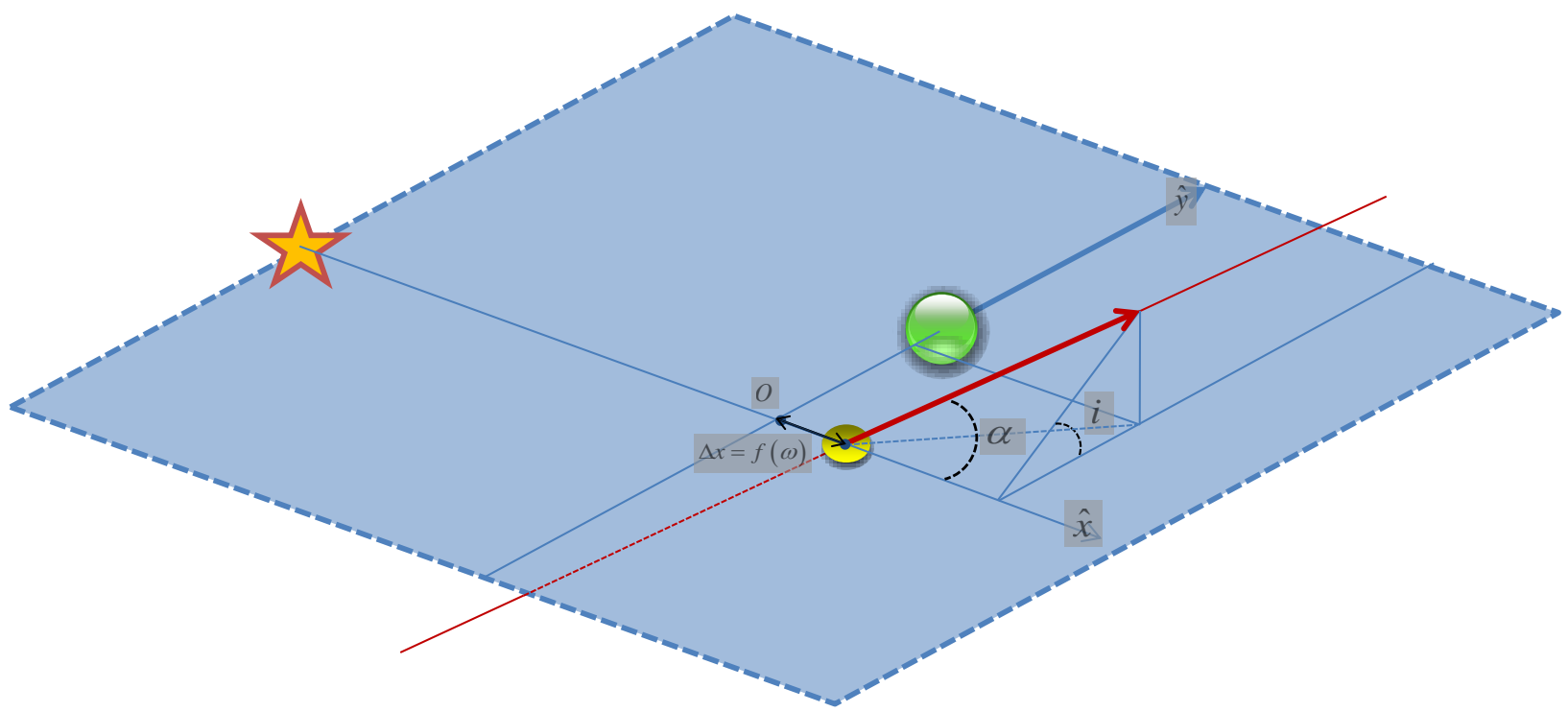

Figure 6. Set of coordinates used to compute Eq.(24).

Close to the values of $\omega_{\text {MOIDO }}$, the variation of MOID as a function of periapsis argument can be approximated linearly ${ }^{12,14}$. With the axis shown in Figure 6, the motion of the Earth and the asteroid can be well described using a linear approximation of the Keplerian velocities of the two objects for true anomaly $\theta_{\text {enc }}$. This defines two straight line trajectories, and thus, the minimum distance between these two linear trajectories can be found. The minimum distance can then be written as an explicit function of $\Delta x$ (i.e., distance between the centre of the coordinates described in Figure 6 and the point at which the asteroid crosses the Earth orbital plane), which can also be described as a linear function of the argument of the periapsis $\omega$. Finally, an expression such as:

$$
M O I D=\frac{\min \left[\left|\omega_{\text {MOID }}-\omega\right|\right]}{\sqrt{\left(\frac{1}{\sin (i)}\right)^{2}+\tan ^{2}(\alpha)}}
$$

yields an approximate value of the MOID distance. The expression min[|.|] denotes the minimum value of the absolute differences with any of the angles $\omega_{\text {MOID } 0}$ and the tangent of the flight path angle can be calculated as:

$$
\tan (\alpha)=\frac{p}{\sqrt{e^{2}-(p-1)^{2}}}
$$

For a complete derivation of a similar formulae, the reader can refer to Opik's work ${ }^{12}$ or alternatively to Bonanno's work ${ }^{14}$. Note that Eq. (24) is valid only for values of $\omega$ close to any of the values of $\omega_{\text {MOID } 0}$ from equation (23). Figure 7 show, as example, the evolution of the MOID distance as a function of periapsis argument for the elliptic orbit plotted in Figure 5 (i.e., a $=1.1 \mathrm{AU}, \mathrm{e}=0.8, \mathrm{i}=30^{\circ}$ ). The figure compares the results of MOID calculated by means of Eq.(24) with the results of a numerical algorithm that finds MOID by minimizing the distance between two positions defined by the true anomaly of each orbit. As it can be seen, Eq.(24) yields a very good approximation of the real MOID when MOID is small. Clearly, the error committed by this formulation increases for very low inclinations and very low eccentricities, but it is still tolerable for inclinations of 0.1 degree and eccentricities of 0.01 . 


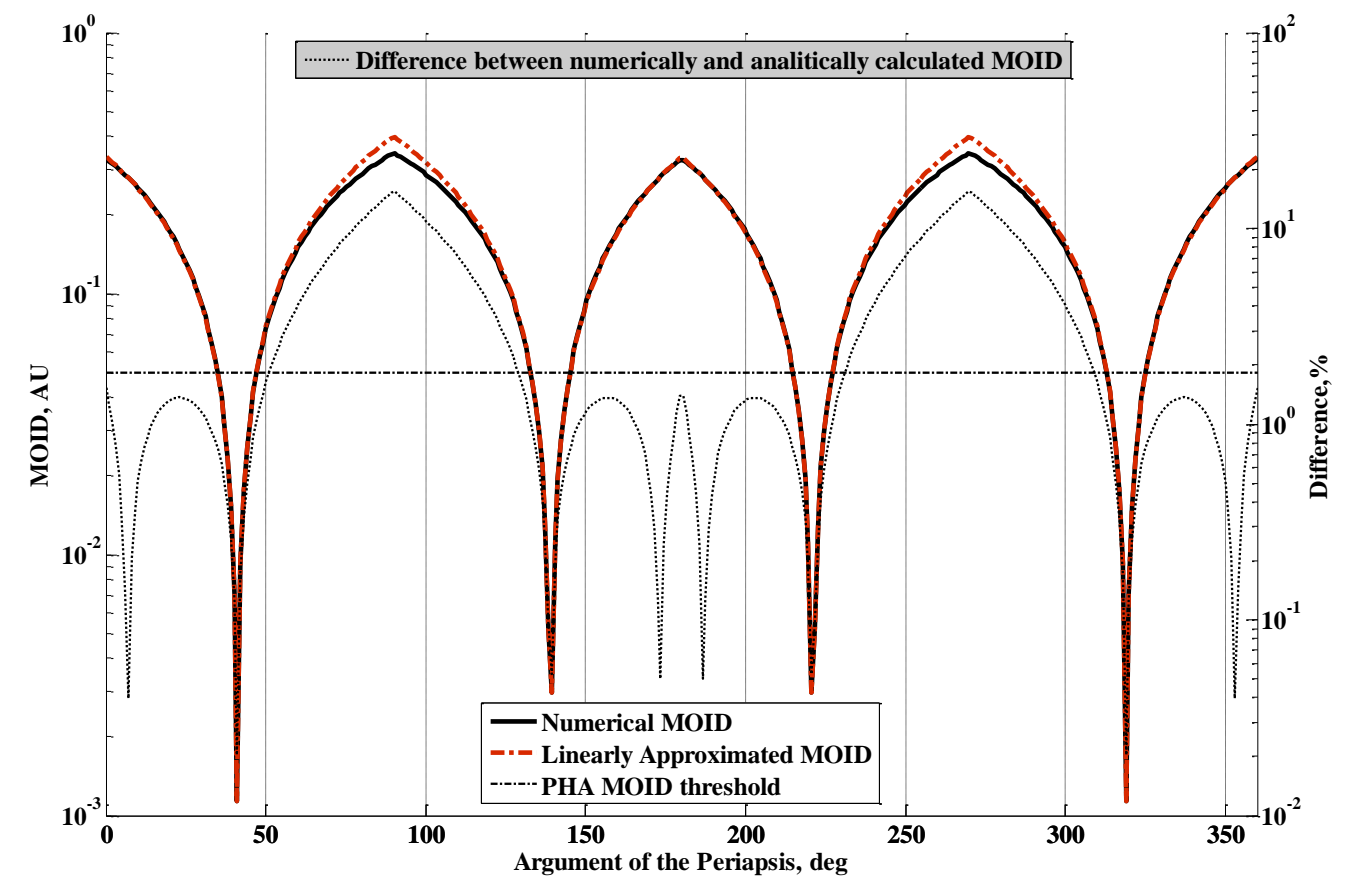

Figure 7. Comparison between the analytical and numerical approaches to compute MOID.

\section{Capture at MOID point}

Once it has been shown that the analytical approximation of MOID is a reliable way of assessing the distance between two orbits, we can define the maximum MOID at which the capture of an object is possible given a limiting $\Delta v$ budget. Eq.(16), in section III.A.2, defined the required Earth capture maneuver $\Delta v_{\text {cap }}$ as a function of the hyperbolic excess velocity $v_{\infty}$ and the pericenter altitude $r_{p}$. The latter can also be expressed as an explicit function of the hyperbolic velocity $v_{\infty}$ and the impulsive maneuver:

$$
r_{p}=\frac{8 \mu_{\oplus} \Delta v_{c a p}^{2}}{\left(v_{\infty}^{2}-\Delta v_{c a p}^{2}\right)^{2}}
$$

Since equation (26) refers to non-coplanar asteroids, the hyperbolic velocity $v_{\infty}$ needs to be calculated as:

$$
v_{\infty}^{2}=\mu_{\text {Sun }}(3-1 / a-2 \sqrt{p} \cdot \cos (i))
$$

This expression can be derived by noticing that the relative velocity at the encounter for a non-coplanar asteroid can be expressed as $\left(v_{r}^{e n c}, v_{n}^{e n c} \cdot \cos (i)-\omega_{\oplus} \cdot r_{e n c}, v_{n}^{e n c} \cdot \sin (i)\right)$.

Finally, in order to know the maximum MOID at which a direct capture is possible, the distance $r_{p}$ needs to be corrected by the hyperbolic factor, i.e., factor that accounts for the gravitational pull of the Earth during the asteroid's final approach to the Earth. This results on:

$$
\mathrm{MOID}_{c a p}=r_{p} \sqrt{1+\frac{2 \mu_{\oplus}}{r_{p} v_{\infty}}}
$$

Note that if the perigee altitude resultant from Eq.(26) is smaller than the radius of the Earth would mean that the capture of that particular body is not feasible under that particular $\Delta v$ threshold used. In fact, the feasible limit for a fly-by was set to $200 \mathrm{~km}$ altitude from the surface of the Earth, also to account for the Earth atmosphere.

\section{Fraction of capturable asteroids}

The previous section provided the means of calculating the MOID at which capture is possible as a function of $\Delta v_{\text {cap }}$. Using the linearly approximated MOID in Eq.(24), we can see that within a distance $\Delta \omega$ of $\omega_{\text {MOIDo }}$ such as: 


$$
\Delta \omega=\operatorname{MOID}_{c a p} \cdot \sqrt{\left(\frac{1}{\sin (i)}\right)^{2}+\tan ^{2}(\alpha)}
$$

a direct capture of the asteroid is possible, since the minimum orbital distance is ensured to be smaller than MOID $_{\text {cap }}$, and thus, the capture impulse should be smaller than $\Delta v_{\text {cap }}$. One may think then that the total range at the neighborhood of $\omega_{\text {MOID } 0}$ is $2 \Delta \omega$, and since there are 4 different $\omega_{\text {MOID } 0}$, the total range of $\omega$ at which capture is possible should be $8 \Delta \omega$. This is generally correct, but attention must be pay when overlapping of ranges occurs. If the semilatus rectum $p$ is close two 1 , the values $\theta_{e n c}$ and $\pi$ - $\theta_{e n c}$ are also close and their ranges $\left(\theta_{e n c} \pm \Delta \omega\right.$ and $\pi$ $\left.\theta_{e n c} \pm \Delta \omega\right)$ may overlap, a correction is applied in those cases.

The fraction of asteroids with given $\{a, e, i\}$ that can be captured with a given $\Delta v$ budget is then:

$$
f(a, e, i)=\frac{8 \Delta \omega(a, e, i)}{2 \cdot \pi}
$$

without overlap correction. Fraction $f$ provides the portion of material with Keplerian elements $\{a, e, i\}$ that could be capture with a single maneuver ( $\left.\leq \Delta v_{\text {cap }}\right)$ at the Earth. Capture of asteroid material by means of only one impulse would simplify considerably the engineering challenges of implementing the two-impulse transfer, described in section III.A, since this type of transfer requires of a spacecraft to be sent to deep-space to perform a change of plane.

\section{Keplerian Feasible Regions}

The capture feasible regions using one-impulse transfers in the $\{a, e\}$ subspace are the same than in section III.A.3. The only difference between the feasible volume $\{a, e, i\}$ of the two-impulse and the one-impulse model lies in the inclination dimension. Since no change of inclination is required, the maximum inclination from which asteroids can be captured is greatly increased. The limit threshold can be computed by realizing that $v_{\infty}^{2}$ calculated as in Eq.(27) must be equal to $v_{\infty}^{2}$ calculated as in Eq.(19), thus:

$$
i_{\max }(a, e, \Delta v)=\cos ^{-1}\left(\frac{1}{2 \sqrt{p}}\left(3-1 / a-\frac{v_{\infty}^{2}}{\mu_{\text {sun }}}\right)\right) \text { where } v_{\infty}^{2} \equiv\left(\Delta v+\sqrt{\frac{2 \mu_{\oplus}}{r_{p}}}\right)^{2}-\frac{2 \mu_{\oplus}}{r_{p}}
$$

where $r_{p}=r_{\oplus}+200 \mathrm{~km}$ is the lower limit for an Earth flyby. Note that, unlike the two-impulse transfer model, the single-impulse transfer is not able to capture all the mass inside the capture feasible region, but only a fraction $f$ (Eq.(30)) of the total mass.

\section{Three-impulse method}

Important enhancement of the accessible Keplerian space can be achieved by adding a further maneuver to the set of impulses described as a two-impulse transfer in Section III.A (i.e., change of plane and Earth capture). Figure 8 show the four cases for which three-impulse transfers are advantageous. Clearly, if more complex transfers are considered, the population of non-Earth crossing asteroids can now be accessed for exploitation, in particular Interior-to-Earth-orbit asteroids (IEOs) and Amor class asteroids could potentially be captured. Also, for asteroids that are already Earth-crossing, a small intermediate maneuver may reduce the necessary $\Delta v$ at the Earth orbital insertion.

A well known technique to reduce the $\Delta v$ requirements for both planetary escape and orbital insertion is the $v_{\infty}{ }^{-}$ leveraging maneuver ${ }^{8}$. In the case of an Earth-crossing asteroid following the sequence of maneuvers described in section III.A, a small $\Delta v_{\mathrm{lev}}$ impulse can be added during the coplanar transfer phase. This maneuver aims to change the conditions of the Earth encounter so that the required $\Delta v_{\text {cap }}$ is substantially smaller. This is $\Delta v_{\text {cap }}^{1}+\Delta v_{\text {lev }}<\Delta v_{\text {cap }}^{0}$, where $\Delta v^{0}$ cap refers to the capture as described in Section III.A, while $\Delta v^{1}$ cap refers to the capture with the orbital geometry resultant after the maneuver $\Delta v_{\text {lev }}$. Figure 3-C and 3-D illustrates the general geometric change of $v_{\infty}{ }^{-}$ leveraging maneuvers. Figure 3-A and 3-B shows the new accessible asteroid orbits. 

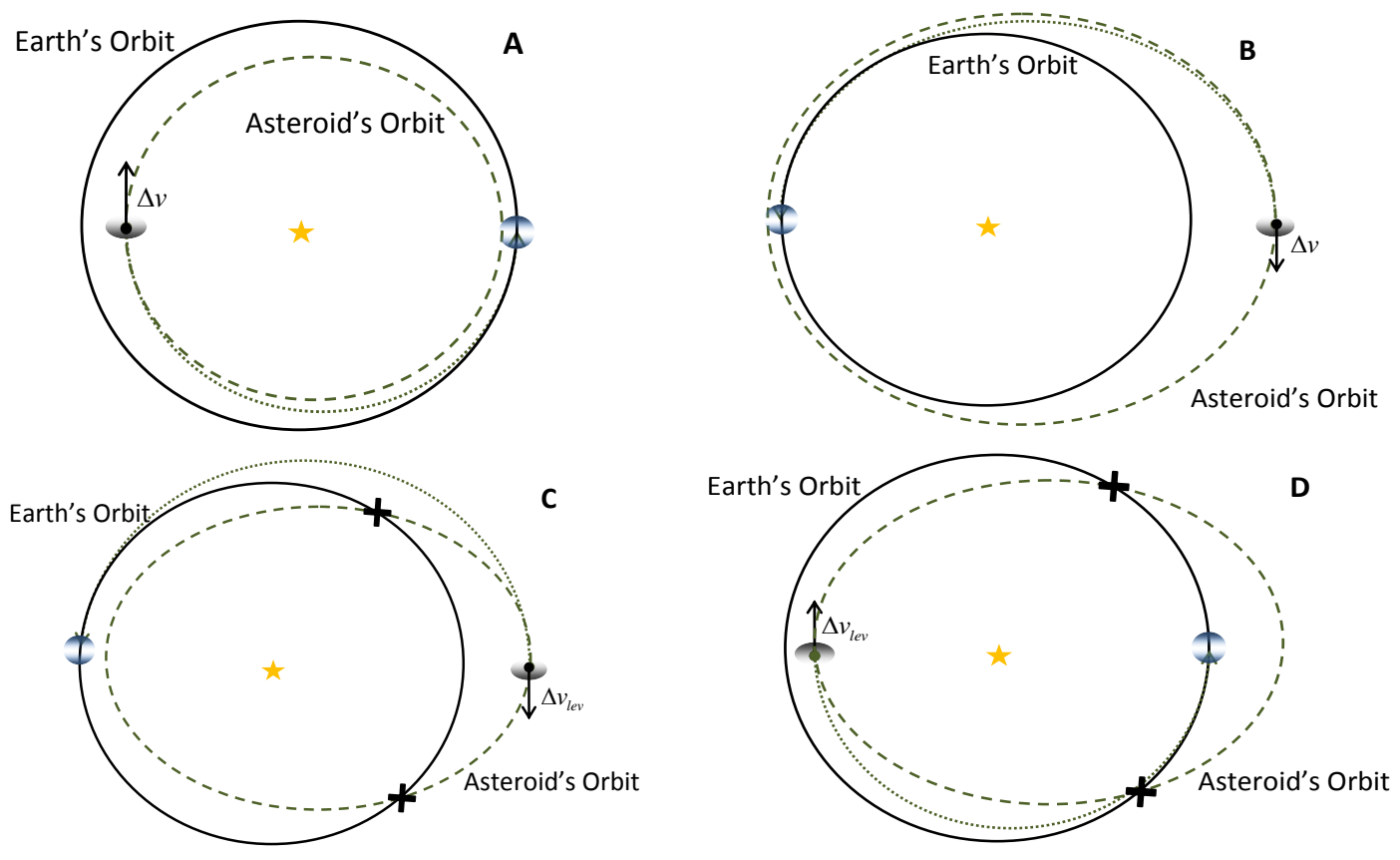

Figure 8. Orbital geometry of Earth transfers and the $\mathbf{v}_{\infty}$-leveraging maneuver. Figure $A$ and $B$ show the Earth transfer for Interior-to-Earth-orbit asteroids (IEO) and Amor class asteroids respectively. Figure $C$ and $D$ show an external and internal $v_{\infty}$-leveraging maneuver, respectively.

Looking at Eq.(16) one can see that the lower the relative velocity at encounter $v_{\infty}$, the lower the impulse required for Earth capture. We can also reckon by looking at Eq.(18) that for a fixed semimajor axis, the lower would be the eccentricity, the lower also the relative velocity. If the eccentricity is too low no intersection with the Earth orbit exists, thus the optimal encounter geometry will always be at the line of apsides of the asteroid. Hence, if IEOs or Amor asteroids are transfer to the Earth, the optimal transfer should be such that the encounter occurs at the aphelion or perihelion respectively, and the transfer impulse should then be located at the opposite apsidal point (see Figure 3-A and 3-B). Equation (32) computes the transfer $\Delta v$ by comparing the vis-viva equation of the instant before and after the impulse for both transfer types, i.e., A and B.

$$
\begin{array}{cc}
\begin{array}{c}
\text { Amor Type } \\
r_{\text {man }}=r_{a}=a(1+e)
\end{array} & \text { IEO } \\
a_{\text {trans }}=\left(r_{a}+1\right) / 2 & r_{\text {man }}=r_{p}=a(1-e) \\
& a_{\text {trans }}=\left(r_{p}+1\right) / 2
\end{array}
$$

Similarly for Earth-crossing asteroids, the optimal point to provide an impulse maneuver that changes the asteroid relative velocity at Earth encounter is also one of the apsidal points. Thus, the $v_{\infty}$-leveraging maneuver can be completely defined by the distance from the sun of the apsidal point where the maneuver is applied $\left(r_{a}\right.$ or $\left.r_{p}\right)$, the semimajor axis $a$ of the initial orbit and the true anomaly of the encounter with the Earth $\theta_{\text {enc }}$ (see Eq.(33)). 


$$
\begin{array}{cr}
\begin{array}{c}
\text { Apohelion maneuver } \\
r_{\text {man }}=r_{a}
\end{array} & \begin{array}{r}
\text { Perihelion maneuver } \\
r_{\text {man }}=r_{p}
\end{array} \\
e_{\text {trans }}=\frac{r_{a}}{a_{\text {trans }}}-1 & e_{\text {trans }}=1-\frac{r_{p}}{a_{\text {trans }}} \\
a_{\text {trans }}=\frac{r_{a}^{2}+r_{a} \cdot \cos \left(\theta_{\text {enc }}\right)}{2 r_{a}-1+\cos \left(\theta_{\text {enc }}\right)} & a_{\text {trans }}=\frac{r_{p}^{2}-r_{p} \cdot \cos \left(\theta_{\text {enc }}\right)}{2 r_{p}-1-\cos \left(\theta_{\text {enc }}\right)} \\
\Delta v_{\text {lev }}=\sqrt{\mu_{\text {sun }}}\left|\sqrt{\frac{2}{r_{\text {man }}}-\frac{1}{a_{\text {trans }}}}-\sqrt{\frac{2}{r_{\text {man }}}-\frac{1}{a}}\right|
\end{array}
$$

\section{Perihelion maneuver}

Equation (33), together with Eq.(16), can be optimized to find the optimal encounter angle $\theta_{\text {enc }}$ that minimizes the most the cost of the coplanar maneuvers $\Delta v_{\text {lev }}+\Delta v_{\text {cap }}$. The result of the optimization shows that whenever the $v_{\infty}{ }^{-}$ leveraging strategy is able to reduce the cost of the capture, then the optimal encounter angle $\theta_{\text {enc }}$ is always one of the apsidal points (either $\pi$ or 0 rads). There is also a region for which the $v_{\infty}$-leveraging is not providing any improvement to the total capture $\Delta v$ budget. Figure 9 shows the regions in the Keplerian plane $\{a, e\}$ in which the $v_{\infty}$-leveraging strategy is advantageous, as well as the approximate reduction factor of $\Delta v_{\text {lev }}+\Delta v_{\text {cap }}$ versus a single $\Delta v_{\text {cap }}$. Also the area of feasible capture for a two-impulse transfer and $\Delta v_{\text {threshold }}$ of $2.37 \mathrm{~km} / \mathrm{s}$ is superposed in the figure for comparison purposes.

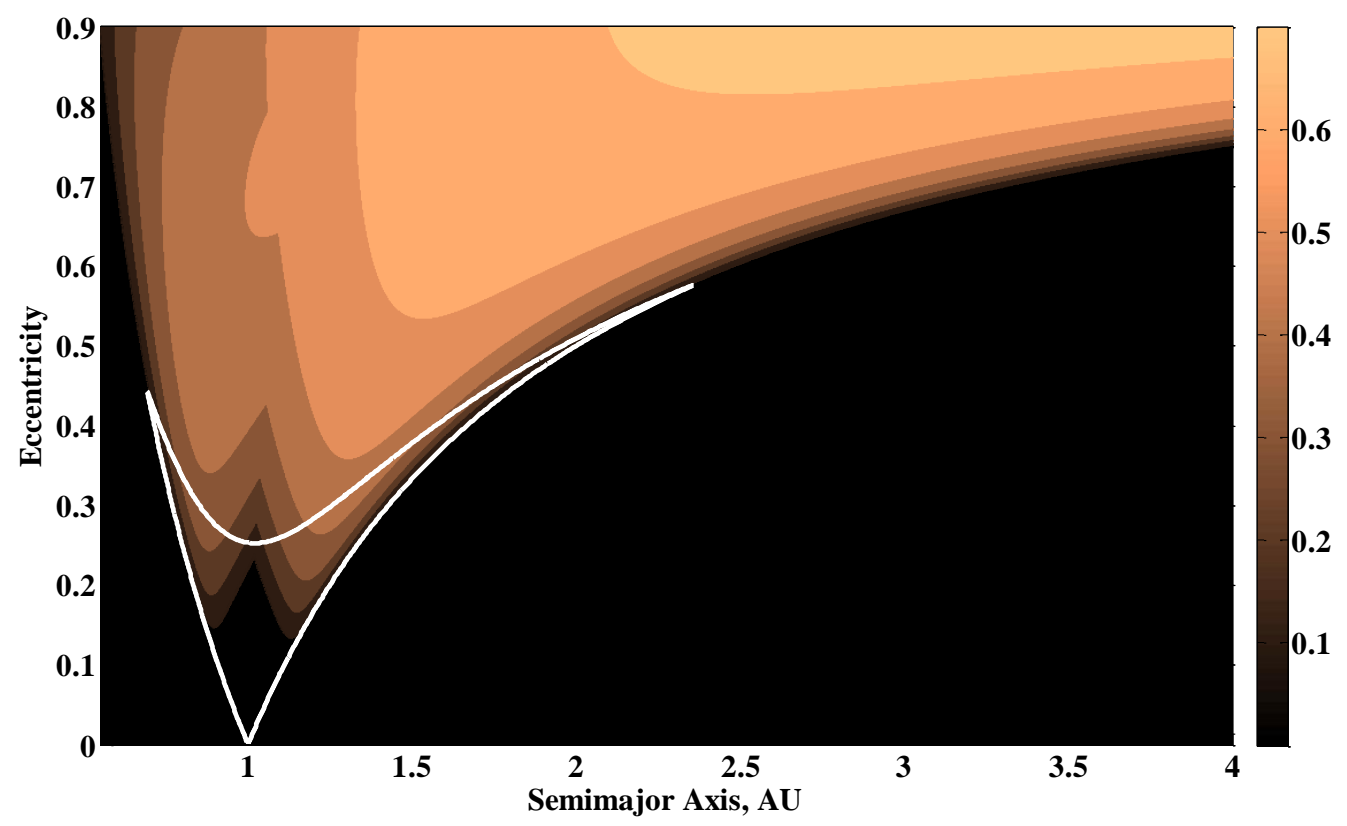

Figure 9. $\{a, e\}$ regions where $v_{\infty}$-leveraging strategy is of advantage for coplanar Earth-crosser asteroids. The color scale shows the fraction of $\Delta v$ saving. Also superposed in the figure is the feasible capture region for a twoimpulse transfer with $\Delta v_{\text {threshold }}$ of $2.37 \mathrm{~km} / \mathrm{s}$.

\section{Keplerian Feasible Regions}

The three-impulse transfer method described here relies on some numerical techniques, such as the numerical optimization of equation (33). Thus, no analytical formulae were found to compute the limits of the feasible region, but a numerical root finding approach was used instead. Figure 10 shows the $\{a, e\}$ Keplerian feasible region for asteroid material exploitation using the three-impulse model and a $\Delta v$ budget of $2.37 \mathrm{~km} / \mathrm{s}$. It is rather clear the gain on feasible area by using the three-impulse approach with a Moon's escape velocity $\Delta v$ budget. Also, as in Figure 4 , the almost 5,000 surveyed asteroids have been superimposed in the figure (small crosses represent asteroids with $\{a, e, i\}$ inside the feasible volume, tiny dots represent otherwise). It can be noted that there has been an increase of crosses with respect Figure 4. In particular, while Figure 4 was showing that 87 currently known asteroids could be 
transfer to Earth orbit with energy lower than the required to escape the Moon's gravity well, Figure 10 shows a total of 214 asteroids.

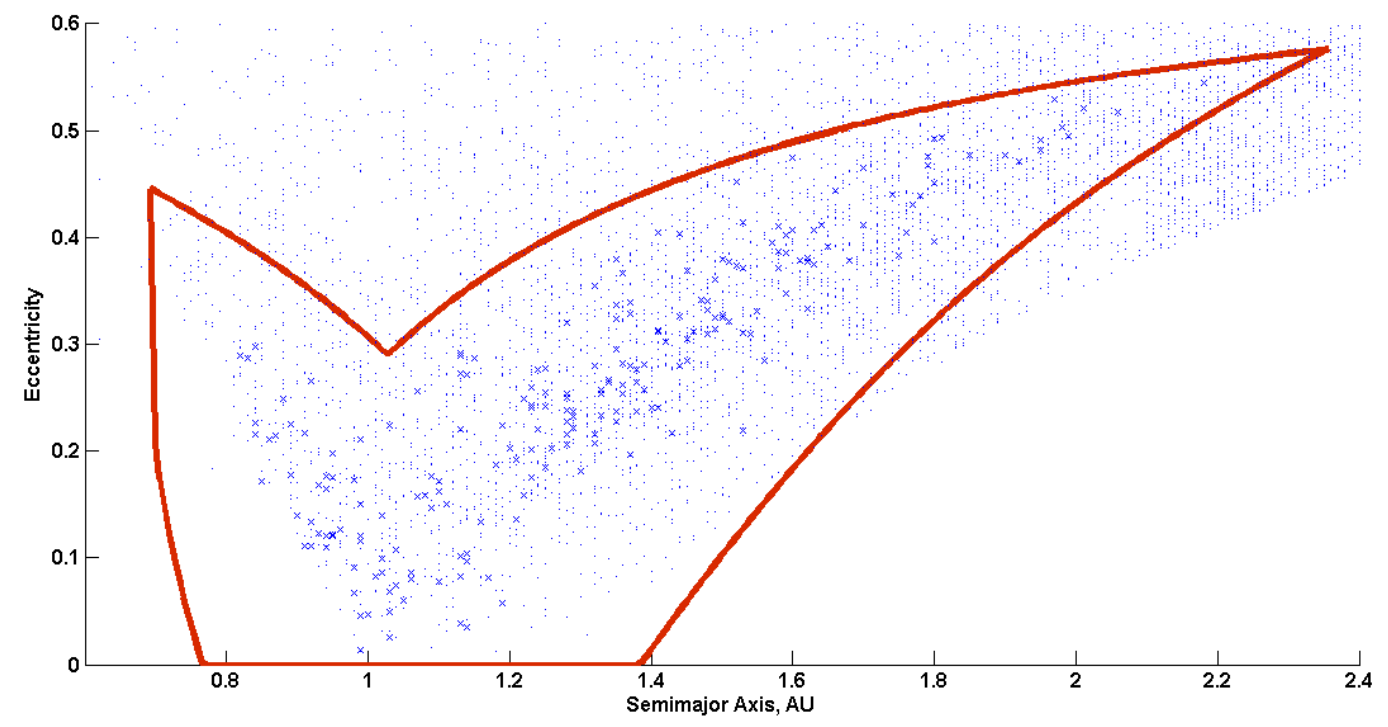

Figure 10. Three-impulse transfer Keplerian \{a,e\} space reached by a maneuver of $2.37 \mathrm{~km} / \mathrm{s}$ (i.e., Moon's escape velocity). Superimposed are all near Earth asteroids known within the \{a,e\} space as of April 2010.

\section{Phasing maneuver}

Previous sections have assumed that if the orbital intersection existed, then the asteroid would eventually meet the Earth. This statement may be true if the time available to transfer the asteroid is not constrained, but for realistic scenarios this does not occur. Therefore, some analysis on the cost of the maneuvering necessary to ensure the encounter opportunity must be performed.

Thus for a more realistic transfer scenario, in which the orbital phasing is also considered, an additional impulsive maneuver may be necessary in order to provide the right phasing to the asteroid. This maneuver is generally small and must be provided as early as possible, so that the secular effect due to the change in period yields the orbital drift necessary for the asteroid to be at the Earth orbital crossing point at the required time. Hence, if only secular effects are considered ${ }^{15-16}$, which is regarded as a good approximation for the level of accuracy intended in this paper, the phasing maneuver should correct the difference in mean anomaly $\Delta \mathrm{M}$ that exists for the intended encounter (see Figure 3). This is expressed as:

$$
\Delta M=\Delta n \cdot\left(t_{e}-t_{m}\right)
$$

where $\Delta n$ is the change of mean motion of the asteroid due to the phasing maneuver and $\left(t_{e}-t_{m}\right)$ is the time-span between the maneuver $\left(t_{m}\right)$ and the encounter (i.e. time at which the Earth is at the crossing point $t_{e}$ ). The change in mean motion of the asteroid can be defined as:

$$
\Delta n=\left(\sqrt{\frac{\mu_{\text {Sun }}}{(a+\delta a)^{3}}}-\sqrt{\frac{\mu_{\text {Sun }}}{a^{3}}}\right)
$$

where $\delta a$ is the change of semi-major axis of the asteroid due to the impulsive maneuver. Using the Gauss planetary equations ${ }^{17}, \delta a$ can be expressed as:

$$
\delta a=\frac{2 a^{2} v_{0}}{\mu_{\text {Sun }}} \delta v_{t}
$$

where $\delta v_{t}$ is the tangential component of the impulsive maneuver and $v_{0}$ is the orbital velocity at the point at which the impulsive maneuver is applied. Eq.(36) seems to indicate that the optimal position for a phasing maneuver is the periapsis, since this is the point at which the orbital velocity $v_{0}$ is maximum. This is generally true, except for cases in which the term $\left(t_{e}-t_{m}\right)$ of equation (34) drives the optimality of the phasing maneuver.

Finally, rearranging Eq.(34), (35) and (36), the phasing maneuver necessary to drift the asteroid a $\Delta \mathrm{M}$ angular position at time $t_{e}$, given a impulsive maneuver at time $t_{m}$, can be expressed as: 


$$
\delta v_{t}=\frac{\mu_{\text {Sun }}}{2 a^{2} v_{0}}\left(\left(\frac{\mu_{\text {Sun }}}{\left(\frac{\Delta M}{\left(t_{e}-t_{m}\right)}+\sqrt{\frac{\mu_{\text {Sun }}}{a^{3}}}\right)^{2}}\right)^{1 / 3}-a\right)
$$

which provides a good estimation of the cost of the encounter. Note that this implies that the optimal phasing maneuver has only tangential impulse.

Considering an Earth-asteroid configuration such as in Figure 3, an algorithm was implemented that computes the fraction of mean anomalies inside the asteroid orbital path that can be phased with the Earth with a $\delta v_{t}$ smaller than a given threshold. The algorithm requires as an input the $\Delta \mathrm{M}$ at a given time $t_{e}$ at which the Earth is assumed to be in the crossing point from which $\Delta \mathrm{M}$ is measured. Also a time constraint needs to be specified, which defines the maximum allowed maneuver time $t_{m}^{\max }$. Then, the algorithm computes the $\delta v_{t}$ necessary to cancel not only the $\Delta \mathrm{M}$ gap at time $t_{e}$, but also all other possible encounters opportunities, which are defined by the times at which the Earth is at the crossing points during the time-span available. For each possible encounter two maneuver times are considered; the first available periapsis passage and the $t_{m}^{\max }$. This procedure is repeated for thousand different angular positions $\Delta \mathrm{M}$ at $t_{e}$, from which then the fraction of the orbit that can be phased under a $\Delta v$ limit is calculated.

\section{Asteroid Exploitable Mass}

Transfers and asteroid population model can finally be set together in order to estimate the available material that could be exploited by future space missions. The total available material will be mapped as a function of the limiting $\Delta v$ budget, and as described in section III, once a $\Delta v$ threshold has been fixed, the Keplerian regions of feasible capture can be calculated for each transfer type. These regions delimit the set of $\{a, e, i\}$ from which an asteroid could be transported to a weakly-bound Earth orbit with a transfer requiring a total $\Delta v$ lower than a fixed threshold. The fraction of the near-Earth object population that falls into this feasible region can be calculated by integrating Eq.(6) over the entire feasible volume. The integration limits of Eq.(6) were defined for each particular transfer model as functions of $\Delta v_{\text {threshold, }}$, semi-major axis $a$ and eccentricity $e$ :

$$
\begin{aligned}
& a_{\min }\left(\Delta v_{\text {threshold }}\right) \geq a \leq a_{\max }\left(\Delta v_{\text {threshold }}\right) \\
& e_{\min }\left(a, \Delta v_{\text {threshold }}\right) \geq e \leq e_{\max }\left(a, \Delta v_{\text {threshold }}\right) \\
& i_{\min }\left(a, e, \Delta v_{\text {threshold }}\right) \geq i \leq i_{\max }\left(a, e, \Delta v_{\text {threshold }}\right)
\end{aligned}
$$

For the free-phase two-impulse and three-impulse transfer, any asteroid within the feasible capture region could, in principle, be capture (or its material exploited). On the other hand, the one-impulse is a special case in which only a fraction $f$ (see Eq.(30)) of asteroids at each $\{a, e, i\}$ point will have the conditions necessary to ensure a capture bellow the $\Delta v$ threshold. Thus, in this case, the integration required is:

$$
P=\int_{a_{\min }}^{a_{\max }} \int_{e_{\min }}^{e_{\max }} \int_{i_{\min }}^{i_{\max }} \rho(a, e, i) \cdot f(a, e, i) \cdot d i \cdot d e \cdot d a
$$

When $\mathrm{P}$ is known, the total mass of near Earth objects is calculated by means of Eq.(5), where a range of diameters need to be specified. By multiplying the probability $\mathrm{P}$ by this total mass of NEA within the given range of object size, one obtains the total asteroid mass that could be theoretically captured and exploited given a $\Delta v$ budget.

\section{A. Total Available Mass}

Figure 11 shows the results of integrating the resources available considering all near Earth objects between $32 \mathrm{~km}$ (i.e., largest Near Earth object known today) and 1 meter diameter. Note that the discussion about the minimum object size has been deliberately avoided so far. One may discourse that objects of 1 meter diameter are too small to be worth a space mission to exploit its raw materials, and thus, a larger limit, such as 10 meters or 100 meters diameter should have been chosen instead. These higher limits of minimum asteroid size barely modify the results on Figure 11, at least in a logarithmic scale, since only $0.42 \%$ or $2.28 \%$ of the asteroid mass is made up by objects sized between 1-10 m or 1-100 m, respectively. Hence, since the lower limit of capturable material has limited effect in the results of the paper, the discussion of whether or not is worth exploiting such small objects as $1 \mathrm{~m}$ diameter can be postponed at this point. 


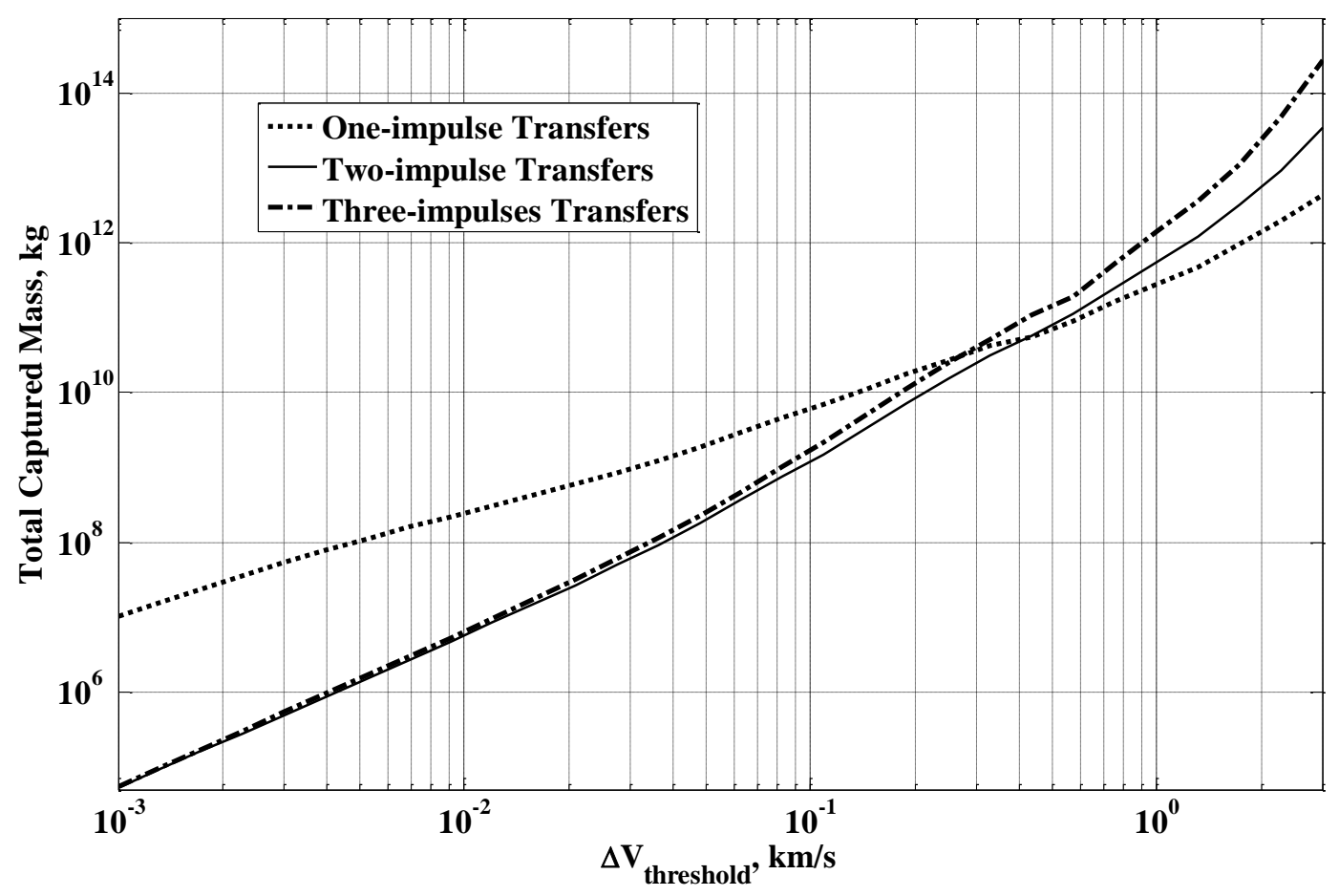

Figure 11. Total asteroid mass available for exploitation of resources as a function of $\Delta \mathrm{v}$ threshold.

A comparison can now be made of the exploitation of lunar and asteroid resources. Figure 11 shows that for $\mathrm{a} \Delta v_{\text {threshold }}$ equal to the Moon's escape velocity (i.e., $2.37 \mathrm{~km} / \mathrm{s}$ ) the total available asteroid material is $2.4 \times 10^{12} \mathrm{~kg}$ if only one maneuver is considered, $1.1 \times 10^{13} \mathrm{~kg}$ for two-impulse transfers and $6.3 \times 10^{13} \mathrm{~kg}$ with three impulses. In addition to the fact that the Moon is believed to be a very resource-poor body ${ }^{18}$, lunar resources would require a minimum $\Delta v$ of $2.37 \mathrm{~km} / \mathrm{s}$ to be extracted from the lunar gravity well. However, as shown in Figure 11, an important aspect of asteroid resources is that they can be exploited at a whole spectrum of $\Delta v$. For example, for a $\Delta v_{\text {thressold }}$ of $100 \mathrm{~m} / \mathrm{s}$ a total mass of $6 \times 10^{9} \mathrm{~kg}$ could be transferred to Earth by using a one-impulse transfer. Considering, for example, that about $10 \%$ of objects are expected to be hydrated carbonaceous asteroids ${ }^{19}$, and that those are believed to have contents of water around $8.5 \%$ of the weight of the asteroid ${ }^{18}$, this $6 \times 10^{9} \mathrm{~kg}$ of material represent, among others exploitable resources, about 25,000 tons of water. All this water should be concentrated in a few of the largest asteroids within the "reachable" region, since in a power law distributed population most of the mass is carried by the largest objects. Note also, that the energetic cost of shipping these 25,000 tons of water to low-Earth Orbit (e.g., $200 \mathrm{~km}$ circular orbit) would be 2.7 times lower than shipping the same the water from the Earth surface. In fact, there should be a total of $4 \times 10^{10}$ tons of water available in near-Earth orbit that could be used in LEO at a lower cost than water from the surface of the Earth.

The transfers models used in this paper provide a conservative scenario for the required $\Delta v$ and so the available asteroid mass found is then a low estimate. More complex trajectories, such as multiple Earth fly-by, lunar gravity assists or manifold dynamics ${ }^{20}$, would be expected to provide a significant increase in captured mass. Note that if an optimized trajectory reduces $\Delta v$ cost in, for example, $33 \%$, the total available mass is increased by a much higher proportion. For example of $100 \mathrm{~m} / \mathrm{s}$ threshold, a $33 \%$ reduction in $\Delta v$ will allow access to the regions reached by 150 $\mathrm{m} / \mathrm{s}$ transfers, which would yield $1.2 \times 10^{10} \mathrm{~kg}$ of mass to be captured, hence a $100 \%$ increase.

Finally, note that one of the underlying assumptions in Bottke et $_{\text {al. }}{ }^{7}$ 's model, and thus also in this paper, is that the orbital distribution is independent of the asteroid size. The orbital distribution described in section II.B assumes that the orbital evolution of asteroids is caused solely by gravitational forces. While this approximation may be more than fine for large objects, for small objects $(<<1 \mathrm{~km})$ the contribution of non-gravitational forces, such as yarkovsky effect, may become an important factor. The minimum object size for which Bottke et $^{\mathrm{al}}{ }^{7}$ model is still a good approximation is not clear ${ }^{21}$, neither the effect of non-gravitational forces in the global evolution of asteroids. These issues shall then be considered in future work. 


\section{B. Maximum and Time Constrained Mass}

As it has been previously remarked, all transfers model were assumed phase free. Thus, it remains to analyze the effects of inducing a correct phasing to asteroid in order to meet the Earth at the orbital crossing points (see Section III.D.). The results shown in Figure 11 assumed that the time available for transferring the asteroid material is sufficiently large so that the phasing maneuver required is negligible, but a more realistic scenario should also considered a maximum time allowed for the transfers described throughout Section III.

In order to include the effects of phasing maneuvers, the integration in Eq.(6) needs now to include a new term to account for the fraction of asteroids that can be phased with the Earth with a $\Delta v$ maneuver lower than the remaining delta-velocity. This remaining $\Delta v$ is, at each $\{a, e, i\}$ point in the Keplerian space, the difference between the $\Delta v$ required for a capture and the given $\Delta v_{\text {threshold. }}$

$$
P_{\Delta t_{\text {transfer }}}=\int_{a_{\min }}^{a_{\max }} \int_{e_{\min }}^{e_{\max }} \int_{i_{\min }}^{i_{\max }} h\left(a, e, \Delta v_{\text {threshold }}-\Delta v_{\text {transfer }}(a, e, i), \Delta t_{\text {transfer }}\right) \cdot \rho(a, e, i) \cdot d i \cdot d e \cdot d a
$$

where $h$ is the fraction of material that can be "phased" with a $\Delta v$ lower that the remaining $\Delta v$ budget, i.e., $\Delta v_{\text {threshold }}-\Delta v_{\text {transfer }}(a, e, i), \Delta v_{\text {transfer }}(a, e, i)$ refers to the cost of the nominal transfer as described in section III and $\Delta t_{\text {transfer }}$ is the limiting time-span for the transfer. A slightly more complicated expression is required to compute $P_{\Delta t_{\text {transfer }}}$ for the case of one-impulse transfers, since the cost of the nominal transfer also depends on the argument of the periapsis, $\Delta v_{\text {transfer }}(a, e, i, \omega)$.Note that, in the one-impulse transfer case, the argument of the periapsis $\omega$ defines the altitude of the fly-by, and thus, strongly influences the cost of the Earth orbital insertion, Eq. (16). In this case, to compute $P_{\Delta t_{\text {transer }}}$, the expression for $f$ in Eq.(38) should be calculated as:

$$
f(a, e, i)=\frac{4 \int_{\omega_{M O D O}-\Delta \omega}^{\omega_{M O I D}+\Delta \omega} h\left(a, e, \Delta v_{\text {threshold }}-\Delta v_{\text {transfer }}(a, e, i, \omega), \Delta t_{\text {transfer }}\right) \cdot d \omega}{2 \cdot \pi}
$$

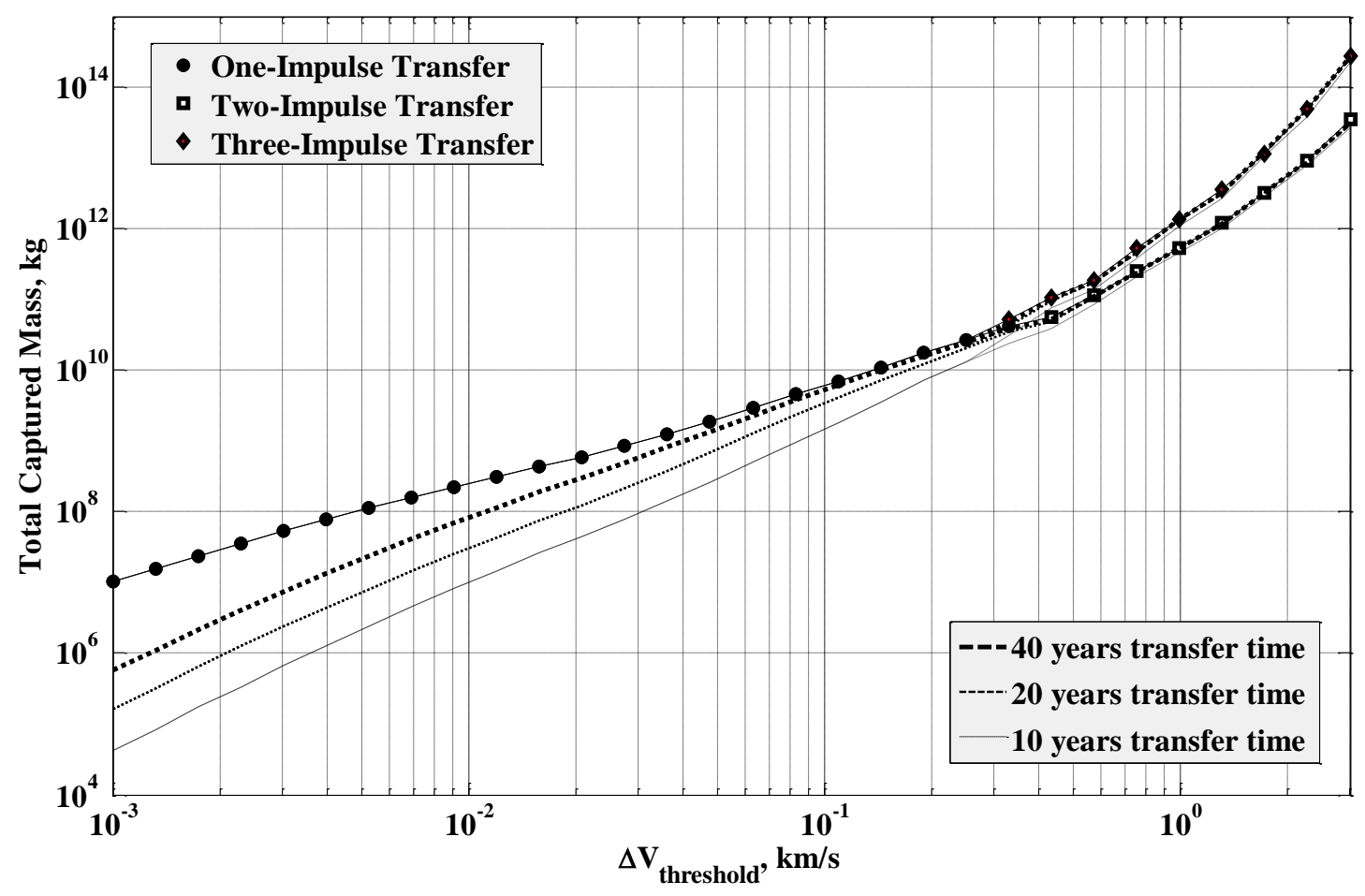

Figure 12. Total captured mass and time constrained exploitable mass.

Figure 12 includes the effect of 40, 20 and 10 years time constraints on the accessibility of asteroid resources. The figure demonstrate how time constraints, although important for the accessibility of resources at very low $\Delta v$ $(<100 \mathrm{~m} / \mathrm{s})$, are not relevant at higher energies. For resources above $1 \mathrm{~km} / \mathrm{s}$ the effect of time constraints are barely detectable. Figure 12 shows only the mass captured with the optimum transfer among the three transfer model presented in the paper. At the low range of $\Delta v(<300 \mathrm{~m} / \mathrm{s})$ a simple one-impulse transfer offer the best option to 
capture near-Earth material, while at higher energies the three-impulse transfer yields better result. Still, the two impulse transfer has been left in the figure when it offers a better option than the one-impulse. The two-impulse transfer, albeit yields lower masses than the three-impulse transfer, offers a conservative estimation of the total mass and a simpler set of maneuvers.

\section{Conclusion}

This paper has shown that the utilization of asteroid resources may be a viable mean of providing substantial mass in Earth orbit for future space ventures. A statistical population of near Earth asteroids has been used, along with a map of the Keplerian orbital element space from which the Earth can be reached under a given series of impulsive maneuvers, to determine the mass available for resources exploitation within a given specific energy. The range of energies for this analysis was selected so that a direct comparison can be made between the cost of exploiting moon and asteroid resources. The results show that there is sufficient asteroid mass in the near-Earth orbital space to justify the exploitation of its resources. The delivery of the resources from targeted asteroids to Earth orbital space for its utilization has been shown to be a more energy efficient approach than lifting the same amount of resources from the surface of the Earth, or even delivering resources from the Moon. Higher energy cost may only be justifiable if the required resource is not available on the Moon. Moreover, the size distribution of objects for near-Earth objects ensures that the amount of exploitable mass is primarily made up of the largest objects within Earth reach. This guarantees that most of the exploitable mass could be successfully harvested by only a few mining or capture missions.

\section{Acknowledgments}

We thank William Bottke for kindly providing us with the NEA distribution data. The work reported was supported by European Research Council grant 227571 (VISIONSPACE).

\section{References}

${ }^{1}$ Mckay, M. F., Mckay, D. S., Duke, M. B., "Space Resources: Scenarios", NASA-SP-509, NASA, Lyndon B. Johnson Space Center. Houston, Texas, 1992.

${ }^{2}$ Chapman, C. R., "The hazard of near-Earth asteroid impacts on Earth", EPSL Frontiers, Vol. 2, 2004, pp. 1-15. Doi:10.1016/j.epsl.2004.03.004

${ }^{3}$ Kawaguchi, J., Fujiwara, A., Uesugi, T., "Hayabusa-Its technology and science accomplishment summary and Hayabusa-2", Acta Astronautica, Vol. 62, No. 10-11, 2008, pp. 639-647. Doi:10.1016/j.actaastro.2008.01.028

${ }^{4}$ Barucci, M., Yoshikawa, M., Michel, P., Kawagushi, J., Yano, H., Brucato, J., Franchi, I., Dotto, E., Fulchignoni, M., Ulamec, S., Marco Polo Science, T., "MARCO POLO: near earth object sample return mission", Experimental Astronomy, Vol. 23, No. 3, 2009, pp. 785-808. Doi:10.1007/s10686-008-9087-8

${ }^{5}$ Sanchez, J. P., Colombo, C., Vasile, M., Radice, G., "Multi-criteria Comparison among Several Mitigation Strategies for Dangerous Near Earth Objects", Journal of Guidance, Control and Dynamics, Vol. 32, No. 1, 2009, pp. $121-142$. Doi:10.2514/1.36774

${ }^{6}$ Stokes, G. H., Yeomans, D. K., Bottke, W. F., Chesley, S. R., Evans, J. B., Gold, R. E., Harris, A. W., Jewitt, D., Kelso, T. S., McMillan, R. S., Spahr, T. B., Worden, P., "Study to Determine the Feasibility of Extending the Search for Near-Earth Objects to Smaller Limiting Diameters", T. Near-Earth Object Science Definition (Ed.), NASA, 2003.

${ }^{7}$ Bottke, W. F., Morbidelli, A., Jedicke, R., Petit, J.-M., Levison, H. F., Michel, P., Metcalfe, T. S., "Debiased Orbital and Absolute Magnitude Distribution of the Near-Earth Objects", Icarus, Vol. 156, No. 2, 2002, pp. $399-433$. Doi:doi:10.1006/icar.2001.6788

${ }^{8}$ Sims, J. A., Longuski, J. M., Staugler, A. J., "Vinf Leveraging for Interplanetary Missions: Multiple-Revolution Orbit Techniques", Journal of Guidance, Control and Dynamics, Vol. 20, No. 3, 1997, pp. 409-415.

${ }^{9}$ Chesley, S. R., Chodas, P. W., Milani, A., Yeomans, D. K., "Quantifying the Risk Posed by Potential Earth Impacts", Icarus, Vol. 159, 2002, pp. 423-432. Doi:10.1006/icar.2002.6910

${ }^{10}$ Steel, D., "Tunguska at 100", Nature, Vol. 453, 2008, pp. 1157-1159. Doi:10.1038/4531157a

${ }^{11}$ Stuart, J. S., "Observational constraints on the number, albedos, size, and impact hazards of the near-Earth asteroids", Massachusetts Institute of Technology, 2003, pp. Thesis (Ph. D.).

${ }^{12}$ Opik, E. J., "Collision Probabilities with the Planets and the Distribution of Interplanetary Matter", Proceedings of the Royal Irish Academy. Section A: Mathematical and Physical Sciences, Vol. 54, 1951/1952, pp. 165-199

${ }^{13}$ Jack, G. H., Goda, M. P., "The Fragmentation of Small Asteroids in the Atmosphere", The Astronomical Journal, Vol. 105, No. 3, 1993, pp. 1114-1144. Doi:10.1086/116499

${ }^{14}$ Bonanno, C., "An analytical approximation for the MOID and its consequences", Astronomy and Astrophysics, Vol. 360, 2000, pp. 411-416.

${ }^{15}$ Scheeres, D. J., Schweickart, R. L., "The Mechanics of Moving Asteroids", Planetary Defense Conference, American Institute of Aeronautics and Astronautics, Orange County, California. 
${ }^{16}$ Izzo, D., "Optimization of Interplanetary Trajectories for Impulsive and Continuous Asteroid Deflection", Journal of Guidance, Control and Dynamics, Vol. 30, No. 2, 2007, pp. 401-408. Doi:10.2514/1.21685

${ }^{17}$ Battin, R. H., Introduction to the Mathematics and Methods of Astrodynamics AIAA Education Series, 1999.

${ }^{18}$ Ross, S. D., "Near-Earth Asteroid Mining", CA: Department of Control and Dynamical Systems, Pasadena, 2001, pp. 1-24.

${ }^{19}$ Bus, S. J., Binzel, R. P., "Phase II of the Small Main-Belt Asteroid Spectroscopic Survey", Icarus, Vol. 158, 2002, pp. 146177. Doi:10.1006/icar.2002.6856

${ }^{20}$ Koon, W. S., Lo, M. W., Marsden, J. E., Ross, S. D., "Dynamical systems, the Three-body Problem and Space Mission Design", International Conference on Differential Equations, 1999.

${ }^{21}$ Veres, P., Jedicke, R., Wainscoat, R., Granvik, M., Chesley, S., Abe, S., Denneau, L., Grav, T., "Detection of Earthimpacting asteroids with the next generation all-sky surveys", Icarus, Vol. 203, No. 2, 2009, pp. $472-485$. Doi:10.1016/j.icarus.2009.05.010 